\title{
Laser photon merging in an electromagnetic field inhomogeneity
}

\author{
Holger Gies, ${ }^{1,2}$ Felix Karbstein, ${ }^{1,2}$ and Rashid Shaisultanov ${ }^{3}$ \\ ${ }^{1}$ Helmholtz-Institut Jena, Fröbelstieg 3, 07743 Jena, Germany \\ ${ }^{2}$ Theoretisch-Physikalisches Institut, Abbe Center of Photonics, \\ Friedrich-Schiller-Universität Jena, Max-Wien-Platz 1, 07743 Jena, Germany \\ ${ }^{3}$ Nazarbayev University, NURIS block 9, 53 Kabanbay Batyr Ave., \\ Astana, 010000, Republic of Kazakhstan
}

(Dated: September 17, 2018)

\begin{abstract}
We study the effect of laser photon merging, or equivalently high harmonic generation, in the quantum vacuum subject to inhomogeneous electromagnetic fields. Such a process is facilitated by the effective nonlinear couplings arising from charged particle-antiparticle fluctuations in the quantum vacuum subject to strong electromagnetic fields. We derive explicit results for general kinematic and polarization configurations involving optical photons. Concentrating on merged photons in reflected channels which are preferable in experiments for reasons of noise suppression, we demonstrate that photon merging is typically dominated by the competing nonlinear process of quantum reflection, though appropriate polarization and signal filtering could specifically search for the merging process. As a byproduct, we devise a novel systematic expansion of the photon polarization tensor in plane wave fields.

PACS numbers: 12.20.Ds, 42.50.Xa, 12.20.Fv
\end{abstract}




\section{INTRODUCTION}

The discovery of quantum vacuum nonlinearities [1 3 ] $]$ under controlled laboratory conditions using real photons or macroscopic electromagnetic fields is a major goal of contemporary strong-field physics. Many proposals rely on a pump-probe scheme, where a well-controlled, say optical, photon beam probes a region of space that is exposed to a strong field ("pump"). A typical example is given by schemes intended to verify vacuum birefringence [4 7] that can be searched for using macroscopic magnetic fields [8, 9] or with the aid of high-intensity lasers [10], see e.g., [11 14] for reviews.

As these setups require techniques such as high-purity ellipsometry [8, 15] to separate the (small) signal from a typically huge background, a recent proposal has focused on a quantumreflection scheme that facilitates a built-in noise suppression [16]. In this scheme, incident probe photons propagate towards a spatially localized field inhomogeneity ("pump"), as, e.g., generated in the focal spots of a high-intensity laser system. Even though the inhomogeneity acts similar to an attractive potential, probe photons can be scattered backwards due to quantum reflection. Looking for reflected photons in the field free region, this scenario inherently allows for a clear geometric separation between signal and background. First estimates of the number of reflected photons attainable in present and near future laser facilities look promising. Figure 1 depicts a typical Feynman diagram contributing to the effect.

As quantum reflection crucially relies on the presence of an inhomogeneous pump field, it belongs to a general class of quantum-induced interference effects [17]19] with the particular property of optimizing the signal-to-noise ratio.

The pump-probe scheme is typically also reflected by the theoretical description, in which the nonlinearities are kept for the pump-probe interaction, but the equations are linearized with respect to the probe propagation. In the present work, we rely again on an optical pump-probe setup which however requires a nonlinear treatment of the probe-field. The idea is to look for laser photon merging in the presence of an electromagnetic field inhomogeneity. This effect resembles the standard nonlinear optical process of second harmonic generation (SHG) - or in general high harmonic generation - with the nonlinear crystal replaced by the quantum vacuum subject to strong electromagnetic fields. Higher harmonic generation in an electromagnetized vacuum has been discussed on the level of the Heisenberg-Euler action in [20 23], see also the discussion in [24], or using the constant-field polarization tensor in [25]. Laser photon merging in proton-laser collisions have been investigated in detail in [26, 27], where a promising scenario has been proposed for a discovery of the merging phenomenon that involves a nowadays conventional optical high-intensity laser at a high-energy proton collider. A related effect is called four-wave mixing for which also a concrete experimental 


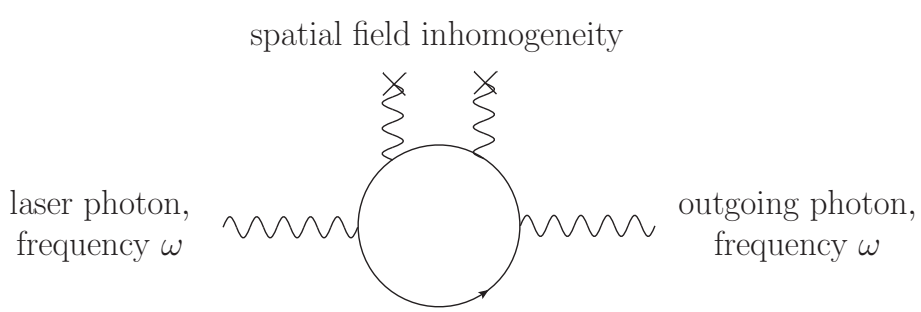

FIG. 1: Typical Feynman diagram contributing to the effect of quantum reflection [16]. For field strengths of the inhomogeneity well below the critical field strength (cf. main text), the leading contribution arises from diagrams with two couplings to the field inhomogeneity. As there is no energy transfer from static fields, the frequencies of the incident and outgoing photons match.

proposal has been explored in [28, 29]. The same underlying quantum vacuum nonlinearity could even be used to radiate photons from the focal spot of a single focused laser beam ("vacuum emission") as proposed in [30]. More generally, frequency mixing induced by quantum vacuum effects has even been suggested as a sensitive probe to search for new hypothetical particles [31].

In the present work, we concentrate on an "all-optical" parameter regime realizable with high-intensity lasers. As the signal is expected to be very small, we again consider specifically the kinematics of the reflection process for an appropriate signal-to-noise reduction. As in [16], we limit ourselves to the study of time-independent field inhomogeneities, such that there is no energy transfer from the field inhomogeneity. Depending on the spatial field inhomogeneity, the propagation direction of the merged photons can differ from that of the incident probe photons. For the specific reflecting kinematic situation, the merged photons can even propagate - somewhat counter-intuitively - into the backward direction. For a straightforward comparison of the signals resulting from quantum reflection [16] and the photon merging scenario of this work, we focus on a one-dimensional magnetic field inhomogeneity. As is shown by an explicit calculation below, our findings confirm the expectation that the merging process for the reflective scenario is dominated by the quantum reflection process for the all-optical parameter regime. Nevertheless, due to a different polarization and frequency dependence, filtering techniques might allow for a discovery of the merging process in this set up as well.

Let us briefly outline the theoretical framework of our study, tailored to an all-optical scenario. Optical lasers operate at frequencies $\omega \sim \mathcal{O}(\mathrm{eV})$ much smaller than the electron mass $m \approx 511 \mathrm{keV}$, constituting a typical scale associated with quantum effects in quantum electrodynamics (QED), such that $\frac{\omega}{m} \ll 1$. Moreover, the maximum field strengths attainable with present and near future laser facilities are small in comparison to the critical field strength $E_{\text {cr }} \equiv \frac{m^{2}}{e}[1]$, i.e., $\left\{\frac{e \mathfrak{E}}{m^{2}}, \frac{e B}{m^{2}}\right\} \ll 1$, with $\mathfrak{E}$ denoting the electric field strength of the probe laser and $B$ the peak magnetic field strength of the spatially localized inhomogeneity. 


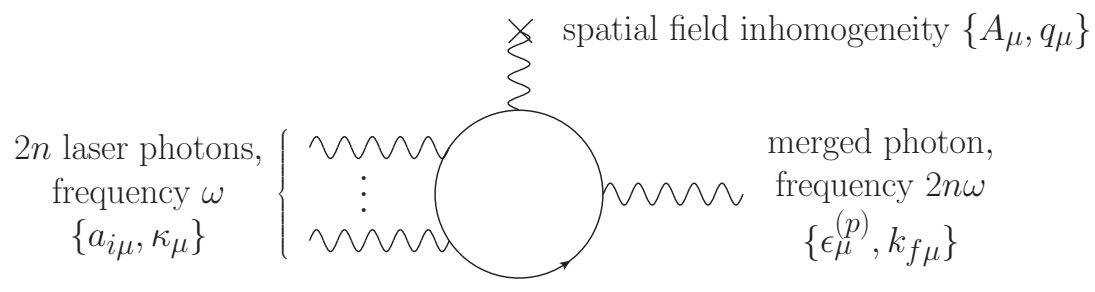

FIG. 2: Cartoon of the photon merging process. In the presence of a stationary but spatially inhomogeneous electromagnetic field $2 n$ laser photons of frequency $\omega$ can merge into a single photon of frequency $2 n \omega$. Depending on the spatial field inhomogeneity, the propagation direction of the merged photons can differ from that of the incident probe photons. In curly braces we introduce our notation for the corresponding fields/polarizations and four-momenta; cf. also Eqs. (11), (26) and (35), as well as Fig. 3 .

Hence, for a given number $2 n, n \in \mathbb{N}$, of probe laser photons of frequency $\omega$ (wavelength $\lambda=\frac{2 \pi}{\omega}$ ), the dominant merging process into a single photon of frequency $2 n \omega$ is expected to arise from an interaction of the type depicted in Fig. 2, exhibiting a single coupling to the (magnetic) field inhomogeneity. Higher order couplings to the field inhomogeneity are strongly suppressed due to the fact that $\frac{e B}{m^{2}} \ll 1$. Furry's theorem (charge conjugation symmetry of QED) dictates the interaction to vanish for any odd number of couplings to the electron-positron loop, which justifies that we have tailored the merging process to $2 n$ laser photons. The dominant contribution in the weak-field limit is expected to arise from the merging of two laser photons, described by Feynman diagrams with four legs (cf. Fig. 2).

A sketch of the geometry of the reflective scenario of the merging process to be investigated in this paper can be found in Fig. 3. Here we already summarize the notation to be introduced and discussed below.

The leading quantum reflection process in the perturbative regime also arises from four leg diagrams (cf. Fig. 1). While quantum reflection necessitates at least two couplings to the field inhomogeneity, photon merging just needs a single coupling to the inhomogeneity. Conversely, quantum reflection can be considered as a two-photon (one incident, one outgoing) process, whereas photon merging involves at least three photons (two incident, one outgoing). From this observation, it can already be anticipated that the dependence of the observables on the various parameters will differ between the two processes.

Notably, the merging process in Fig. 2 can be evaluated straightforwardly, owing to the fact that the photon polarization tensor is explicitly known for generic monochromatic plane wave backgrounds [32, 33]. Interpreting the plane wave background in terms of incident probe photons of frequency $\omega$, the two open legs of the polarization tensor can be identified with the field inhomogeneity and the outgoing merged photon, respectively. The polarization of the incident photons can be controlled by adjusting the polarization of the monochromatic 


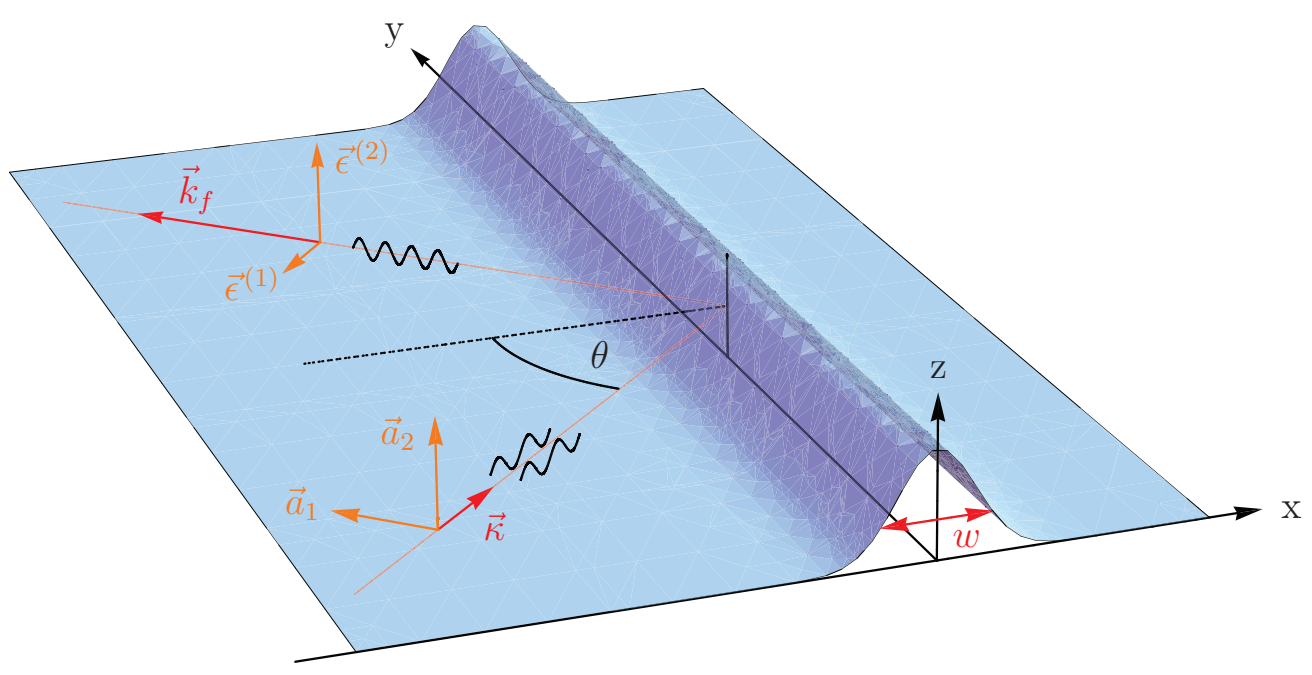

FIG. 3: Schematic depiction of the two-photon merging process. Incident probe photons (wave vector $\vec{\kappa}$, energy $\left.\kappa^{0}=|\vec{\kappa}|=\omega\right)$ hit a one-dimensional field inhomogeneity $\vec{B}(\mathrm{x})=B(\mathrm{x}) \vec{e}_{\mathrm{z}}$ of width $w$ under an angle of $\theta$. Due to nonlinear effective couplings between electromagnetic fields mediated by virtual charged particle fluctuations, the field inhomogeneity can impact incident probe photons to merge and form an outgoing photon (wave-vector $\vec{k}_{f}$ ) of twice the energy of the incident probe photons, i.e., $k_{f}^{0}=\left|\vec{k}_{f}\right|=2 \omega$. Most notably, the inhomogeneity can affect the outgoing merged photons to reverse their momentum component along $\vec{e}_{\mathrm{x}}$ with respect to the incident probe photons. The vectors $\vec{a}_{1}, \vec{a}_{2}$ and $\vec{\epsilon}^{(1)}, \vec{\epsilon}^{(2)}$ span the polarization degrees of freedom of the incident and outgoing photons, respectively. For the depiction we specialized to $\varphi=\varphi^{\prime}=0$ (cf. the main text).

plane wave background.

Our paper is organized as follows: In Sec. [I] we explain in detail the various steps needed to evaluate the photon merging process. A crucial technical step is to find a controlled approximation to the photon polarization tensor in a plane wave background, facilitating an analytical treatment of the photon merging process. Such an approximation, especially suited to the parameters of an all-optical experimental scenario, is derived in Sec. IA Section [III is devoted to the discussion of explicit examples and results. It contains a thorough comparison of the effects of laser photon merging and quantum reflection. We end with conclusions and an outlook in Sec. IV]

\section{CALCULATION}

\section{A. Photon polarization tensor in plane wave field}

We briefly recall and summarize the basic structure of the photon polarization tensor in a generic, elliptically polarized monochromatic plane wave background [32, 33]. The latter 
is parametrized by the following gauge potential in Coulomb-Weyl gauge

$$
\mathcal{A}_{\mu}(x)=\mathfrak{a}_{1} a_{1 \mu} \cos (\kappa x)+\mathfrak{a}_{2} a_{2 \mu} \sin (\kappa x),
$$

with $\mathcal{A}_{0}=0, \kappa^{2}=0$ and $a_{1} \kappa=a_{2} \kappa=a_{1} a_{2}=0$. Moreover, we will use the frequency $\omega \equiv \kappa^{0}$. The four-vectors $a_{i \mu}$ with $i \in\{1,2\}$ are normalized to unity, i.e., $a_{i}^{2}=1$, and the field amplitude is encoded in the coefficients $\mathfrak{a}_{i} \geq 0$. Our metric convention is $g_{\mu \nu}=$ $\operatorname{diag}(-1,+1,+1,+1)$, and we use $c=\hbar=1$.

For the normalized plane wave field strength in momentum space, we introduce $f_{i}^{\mu \nu}=$ $\kappa^{\mu} a_{i}^{\nu}-\kappa^{\nu} a_{i}^{\mu}$. In the following, we will frequently use the shorthand notation $\left(k f_{i}\right)^{\mu}=k_{\nu} f_{i}^{\nu \mu}$.

In momentum space the photon polarization tensor mediates between two four-momenta $k_{1}$ and $k_{2}$. Since the wave (11) is characterized by the single four-momentum $\kappa$ and a change in the incident momentum is determined by an interaction with the wave, the kinematics are such that $k_{2}=k_{1}+C \kappa$, with scalar constant $C$ [32]. Correspondingly, $\kappa k_{2}=\kappa k_{1} \equiv \kappa k$ and also $\left(k_{1} f_{i}\right)^{\mu}=\left(k_{2} f_{i}\right)^{\mu}=\left(k f_{i}\right)^{\mu}$.

Following [32], the associated photon polarization tensor can then be compactly represented as

$$
\Pi^{\mu \nu}\left(k_{1}, k_{2}\right)=c_{1} \Lambda_{1}^{\mu} \Lambda_{2}^{\nu}+c_{2} \Lambda_{2}^{\mu} \Lambda_{1}^{\nu}+c_{3} \Lambda_{1}^{\mu} \Lambda_{1}^{\nu}+c_{4} \Lambda_{2}^{\mu} \Lambda_{2}^{\nu}+c_{5} \Lambda_{3}^{\mu} \Lambda_{4}^{\nu}
$$

with scalar coefficients $c_{j}\left(k_{1}, k_{2}\right), j \in\{1, \ldots, 5\}$. The tensor structure is encoded in products of the normalized four vectors

$$
\begin{aligned}
& \Lambda_{i}^{\mu}=\frac{\left(k f_{i}\right)^{\mu}}{(\kappa k)}=a_{i}^{\mu}-\frac{\left(k a_{i}\right)}{(\kappa k)} \kappa^{\mu} \text { for } i \in\{1,2\}, \\
& \qquad \Lambda_{3}^{\mu}=\frac{\kappa^{\mu} k_{1}^{2}-k_{1}^{\mu}(\kappa k)}{(\kappa k) \sqrt{-k_{1}^{2}}}, \quad \Lambda_{4}^{\mu}=\frac{\kappa^{\mu} k_{2}^{2}-k_{2}^{\mu}(\kappa k)}{(\kappa k) \sqrt{-k_{2}^{2}}},
\end{aligned}
$$

fulfilling $\Lambda_{1}^{2}=\Lambda_{2}^{2}=\Lambda_{3}^{2}=\Lambda_{4}^{2}=1$. This tensor structure guarantees that $\Pi^{\mu \nu}\left(k_{1}, k_{2}\right)$ satisfies the Ward identities $k_{1, \mu} \Pi^{\mu \nu}\left(k_{1}, k_{2}\right)=\Pi^{\mu \nu}\left(k_{1}, k_{2}\right) k_{2, \nu}=0$.

Apart from a trivial overall factor of $\alpha=e^{2} /(4 \pi)$, the coefficients $c_{j}$ depend on the kinematic variables $k_{1}, k_{2}$ and $\kappa$ as well as the electron mass $m$, and account for the entire field strength dependence. The latter dependence is most conveniently expressed in terms of the two invariant intensity parameters $\xi_{i}=\frac{e \mathfrak{a}_{i}}{m}$ with $i \in\{1,2\}$. In Coulomb-Weyl gauge, the amplitude $\mathfrak{a}_{i}$ is intimately related to the amplitude of the associated electric field $\mathfrak{E}_{i}$ via $\mathfrak{a}_{i}=\frac{\mathfrak{E}_{i}}{\omega}$, such that - in terms of parameters directly accessible in the lab - we have $\xi_{i}=\frac{e \mathfrak{E}_{i}}{m \omega}$.

In consequence of Furry's theorem, the field dependence can be encoded in $\xi_{1}^{2}$, $\xi_{2}^{2}$ and $\xi_{1} \xi_{2}$, i.e., combinations even in the charge $e$, only. It is moreover helpful to introduce the dimensionless parameter $\lambda=-\frac{\kappa k}{2 m^{2}}$, parametrizing the relative momenta of the involved 
photons. In summary, the relevant dimensionless parameters for the off-shell polarization tensor in a plane wave field are given by

$$
\xi_{i}=\frac{e \mathfrak{E}_{i}}{m \omega}, \quad \lambda=-\frac{\kappa k}{2 m^{2}}, \quad \frac{k_{1} k_{2}}{4 m^{2}},
$$

where the last parameter characterizes the relative momenta of the in- and outgoing photon legs.

In the following, we are only interested in a situation with actual interactions with the plane wave field (11) and thus omit the zero field contribution in Eq. (2) ${ }^{1}$

The coefficients $c_{j}$ generically decompose into an elastic part characterized by zero momentum exchange with the wave and an inelastic part with finite momentum exchange. The latter part is made up of an infinite number $l \in \mathbb{Z} \backslash\{0\}$ of contributions with momentum transfer $2 \kappa l$ to be associated with the absorption/release of $2 l$ laser photons. Correspondingly, we write

$$
c_{j}=i(2 \pi)^{4} m^{2} \frac{\alpha}{\pi}\left[\delta\left(k_{1}-k_{2}\right) G_{j}^{0}+\sum_{l \in \mathbb{Z} \backslash\{0\}} \delta\left(k_{1}-k_{2}-2 l \kappa\right) G_{j}^{l}\right]
$$

where the dimensionless coefficients $G_{j}^{l}\left(k_{1}, k_{2}\right)$, with $l \in \mathbb{Z}$, are most conveniently represented in terms of double parameter integrals that cannot be tackled analytically in a straightforward way. One of the integrals is over a proper-time type parameter $\rho \in[0, \infty[$, and the other one over an additional parameter $\nu \in[-1,1]$ related to the momentum routing in the loop.

In order to state them most compactly, it is convenient to define

$$
\begin{aligned}
A=\frac{1}{2}\left(1-\frac{\sin ^{2} \rho}{\rho^{2}}\right), \quad A_{0}=\frac{1}{2} \rho\left(\partial_{\rho} A\right), \quad A_{1} & =A+2 A_{0}, \\
z & =\frac{2\left(\xi_{1}^{2}-\xi_{2}^{2}\right)}{|\lambda|\left(1-\nu^{2}\right)} \rho A_{0}, \quad y=\frac{2\left(\xi_{1}^{2}+\xi_{2}^{2}\right)}{|\lambda|\left(1-\nu^{2}\right)} \rho A .
\end{aligned}
$$

Taking these definitions into account, the explicit expressions for $G_{j}^{l}$ read

$$
G_{j}^{l}=\int_{-1}^{1} \mathrm{~d} \nu \int_{0}^{\infty} \frac{\mathrm{d} \rho}{\rho} \mathrm{e}^{-i \phi_{0} \rho} g_{j}^{l} \mathrm{e}^{-i y}
$$

\footnotetext{
${ }^{1}$ More precisely, the coefficients $c_{j}$ provided in the following correspond to the quantity $\Pi^{\mu \nu}(A)-\Pi^{\mu \nu}(A=$ $0)$. The zero field term can be included straightforwardly, noting that $g^{\mu \nu}-\frac{k_{1}^{\mu} k_{1}^{\nu}}{k_{1}^{2}}=\Lambda_{1}^{\mu} \Lambda_{1}^{\nu}+\Lambda_{2}^{\mu} \Lambda_{2}^{\nu}+\Lambda_{3}^{\mu} \Lambda_{3}^{\nu}$.
} 
where

$$
\phi_{0}=\frac{2}{|\lambda|\left(1-\nu^{2}\right)}\left[1-i \epsilon+\frac{k_{1} k_{2}}{4 m^{2}}\left(1-\nu^{2}\right)\right],
$$

with $\epsilon \rightarrow 0^{+}$, and

$$
\begin{aligned}
g_{1}^{l}= & \xi_{1} \xi_{2}\left(2 \operatorname{sign}(\lambda) \frac{1+\nu^{2}}{1-\nu^{2}} \rho A_{0}-A_{1} \frac{l}{z}\right) i^{l} J_{l}(z), \\
g_{2}^{l}= & g_{1}^{l}\left(A_{0} \rightarrow-A_{0}, z \rightarrow z, A_{1} \rightarrow A_{1}\right), \\
g_{3}^{l}= & \left(\xi_{1}^{2} A_{1}-\frac{\xi_{1}^{2}-\xi_{2}^{2}}{1-\nu^{2}} \sin ^{2} \rho\right) i^{l}\left(J_{l}(z)-i J_{l}^{\prime}(z)\right)+\xi_{1}^{2} \frac{1+\nu^{2}}{1-\nu^{2}} \sin ^{2} \rho i^{l} J_{l}(z) \\
& +\frac{1}{4}\left(\frac{k_{1} k_{2}}{m^{2}}-\frac{i|\lambda|\left(1-\nu^{2}\right)}{\rho}\right) i^{l}\left(J_{l}(z)-\delta_{l 0} \mathrm{e}^{i y}\right), \\
g_{4}^{l}= & g_{3}^{l}\left(\xi_{1}^{2} \leftrightarrow \xi_{2}^{2}\right)(-1)^{l}, \\
g_{5}^{l}= & -\frac{\sqrt{k_{1}^{2} k_{2}^{2}}}{4 m^{2}}\left(1-\nu^{2}\right) i^{l}\left(J_{l}(z)-\delta_{l 0} \mathrm{e}^{i y}\right),
\end{aligned}
$$

for $l \in \mathbb{Z}$. Here, $J_{l}(z)$ denotes the Bessel function of the first kind, and $\delta_{l l^{\prime}}$ is the Kronecker delta. Equations (21)-(9) constitute the full expression of the photon polarization tensor in a generic plane wave background of type (11) [32, 33]; see [34] for a more recent derivation and an alternative representation. Noteworthily, whenever one of the momenta $k_{1}$ and $k_{2}$ is on the light cone, i.e., either $k_{1}^{2}=0$ or $k_{2}^{2}=0$, the coefficients $G_{5}^{l}$ vanish for all $l \in \mathbb{Z}$, such that $c_{5}=0$. Except for the zero field contribution (cf. footnote 1), the tensor structure of the photon polarization tensor under these conditions can be written entirely in terms of the four-vectors $\Lambda_{1}^{\mu}$ and $\Lambda_{2}^{\mu}$.

For completeness, note that for a circularly polarized plane wave background, corresponding to the choice of $\xi_{1}=\xi_{2}$, we have $z=0$. Hence, taking into account that $J_{l}(z) \sim z^{|l|}$ [cf. Eq. (13) below], the only nonvanishing contributions (9) are those with $l \in\{0, \pm 1\}$, corresponding to the possibility of an elastic interaction and an interaction involving the emission/absorption of just two photons from the circularly polarized wave. The physical reason for this is that a circularly polarized wave has definite chirality, such that transitions are only possible without a change in the chirality of the incident photon $(l=0)$ or with a reversal of its chirality $(l= \pm 1)[32,33]$.

As the expressions are rather cumbersome, we subsequently aim at an approximation particularly suited for all-optical experiments. Our strategy to achieve this relies on series expansions of the expression $g_{j}^{l} \mathrm{e}^{-i y}$ in the integrand of Eq. (7), such that both integrals can be performed explicitly and handy approximations for the polarization tensor are obtained. Similar expansion strategies have recently also led to new analytical insights into the wellknown polarization tensor for constant fields [35]. 
For this purpose it is particularly helpful to note that $A$ and $A_{0}$ have the following infinite series representations [cf. Eq. (6)],

$$
A=\frac{\rho^{2}}{6} \sum_{n=0}^{\infty} A^{(2 n)} \rho^{2 n}, \quad A_{0}=\frac{\rho^{2}}{6} \sum_{n=0}^{\infty}(1+n) A^{(2 n)} \rho^{2 n}
$$

with $A^{(2 n)}=\frac{3}{2} \frac{(2 i)^{2 n+4}}{(2 n+4) !}$; our definitions are such that $A^{(0)}=1$.

The above series representations suggest to define

$$
\zeta^{ \pm} \equiv \frac{\left(\xi_{1}^{2} \pm \xi_{2}^{2}\right) \rho^{3}}{3|\lambda|\left(1-\nu^{2}\right)}
$$

and to rewrite the quantities $y$ and $z$ as follows

$$
y=\zeta^{+} \sum_{n=0}^{\infty} A^{(2 n)} \rho^{2 n}, \quad z=\zeta^{-} \sum_{n=0}^{\infty}(1+n) A^{(2 n)} \rho^{2 n} .
$$

Another important ingredient in our approach is the series representation of $J_{l}(z)$, which, for $l \in \mathbb{Z}$, reads (cf. formulae 8.404 and 8.440 of [36])

$$
J_{l}(z)=\sum_{j=0}^{\infty} \frac{(-1)^{j}[\operatorname{sign}(l)]^{l}}{j !(|l|+j) !}\left(\frac{z}{2}\right)^{|l|+2 j} \quad \text { for } \quad|\arg (z)|<\pi
$$

where $[\operatorname{sign}(l)]^{l}=1$ for $l=0$ is implicitly understood. Inserting Eq. (12) into Eq. (13), all the Bessel functions occurring in Eq. (9) can be expanded in powers of $\zeta^{-}$and $\rho^{2}$. Analogously, factors of $\mathrm{e}^{-i y}$ can be expanded in powers of $\zeta^{+}$and $\rho^{2}$.

In the following, let us assume that $\left|\frac{k_{1} k_{2}}{4 m^{2}}\right|<1$, which is well compatible with an alloptical experimental scenario. Building on this assumption, and resorting to the identity $\int_{0}^{\infty} \frac{\mathrm{d} \rho}{\rho} \mathrm{e}^{-i \phi_{0} \rho} \rho^{l+1}=l !\left(\frac{-i}{\phi_{0}}\right)^{l+1}$ for $l \in \mathbb{N}_{0}$, we obtain

$$
\int_{0}^{\infty} \frac{\mathrm{d} \rho}{\rho} \mathrm{e}^{-i \phi_{0} \rho} \rho^{l+1}=l !\left(-\frac{i}{2}|\lambda|\left(1-\nu^{2}\right)\right)^{l+1} \sum_{n=0}^{\infty}\left(\begin{array}{c}
n+l \\
n
\end{array}\right)\left(-\frac{k_{1} k_{2}}{4 m^{2}}\left(1-\nu^{2}\right)\right)^{n} .
$$

Having implemented the above expansions, the polarization tensor can formally be written in terms of multiple infinite sums. Noteworthily, all $\nu$ integrals are of the following type

$$
\begin{aligned}
\int_{-1}^{1} \mathrm{~d} \nu\left(1-\nu^{2}\right)^{n} & =\frac{2^{2 n+1}(n !)^{2}}{(2 n+1) !} \\
\int_{-1}^{1} \mathrm{~d} \nu\left(1+\nu^{2}\right)\left(1-\nu^{2}\right)^{n} & =\left(1+\frac{1}{2 n+3}\right) \int_{-1}^{1} \mathrm{~d} \nu\left(1-\nu^{2}\right)^{n}
\end{aligned}
$$


with $n \in \mathbb{N}_{0}$, and can straightforwardly be performed explicitly for each contribution.

Thus, with the collective notation $\xi^{2} \in\left\{\xi_{1}^{2}, \xi_{2}^{2}, \xi_{1} \xi_{2}\right\}$ a generic contribution to the photon polarization tensor reads

$$
\begin{gathered}
\int_{-1}^{1} \mathrm{~d} \nu \int_{0}^{\infty} \frac{\mathrm{d} \rho}{\rho} \mathrm{e}^{-i \phi_{0} \rho}\left(\frac{\xi^{2} \rho^{2}}{6}\right)^{s} \rho^{l}\left(\zeta^{+}\right)^{n}\left(\zeta^{-}\right)^{j}\left\{\begin{array}{c}
1 \\
1-\nu^{2} \\
\frac{1}{1-\nu^{2}} \\
\frac{1+\nu^{2}}{1-\nu^{2}}
\end{array}\right\} \\
=\left(-\frac{2 \xi^{2} \lambda^{2}}{3}\right)^{s}\left(i \frac{2\left(\xi_{1}^{2}+\xi_{2}^{2}\right) \lambda^{2}}{3}\right)^{n}\left(i \frac{2\left(\xi_{1}^{2}-\xi_{2}^{2}\right) \lambda^{2}}{3}\right)^{j}(-2 i|\lambda|)^{l} \\
\times c(n, j, s, l)\left\{\begin{array}{c}
1 \\
1-\frac{1}{4(n+j+s)+2 l+3} \\
1+\frac{1}{2} \frac{1}{2(n+j+s)+l} \\
1+\frac{1}{2(n+j+s)+l}
\end{array}\right\}\left(1+\mathcal{O}\left(\frac{k_{1} k_{2}}{4 m^{2}}\right)+\mathcal{O}\left(\lambda^{2}\right)\right)
\end{gathered}
$$

with integers $\{l, n, j\} \in \mathbb{N}_{0}$ and $s \in\{0,1\}$, fulfilling $l+n+j+s>0$. The components in the columns in braces exhaust all possible types of occurring $\nu$ integrands. The explicit expression for the numeric coefficient in Eq. (16) is

$$
c(n, j, s, l)=\frac{2[3(n+j)+2 s+l-1] !\{[2(n+j+s)+l] !\}^{2}}{[4(n+j+s)+2 l+1] !}
$$

Both integrations can be carried out, and Eq. (16) provides us with the full numeric prefactor for given integers $l, n, j$ and $s$ at leading order in a double expansion in $\left|\frac{k_{1} k_{2}}{4 m^{2}}\right| \ll 1$ and $|\lambda| \ll 1$, both corresponding to a soft-photon limit. Most importantly, the parameters $\xi_{i}$ never come alone but always appear in combination with a factor of $\lambda$. This implies that any perturbative expansion of the photon polarization tensor in plane wave backgrounds which is superficially in powers of $\xi^{2}$ in fact amounts to an expansion in the combined parameter $\xi^{2} \lambda^{2}$. This is of substantial practical relevance, as optical high-intensity lasers are entering the regime $\xi \gg 1$. Still the present expansion remains valid as long as $\xi^{2} \lambda^{2} \ll 1$ which is typically well satisfied for contemporary optical high-intensity lasers. First indications of a larger validity regime of the naive "small- $\xi$ " expansion had already been observed in [27]. Our all-order series expansion of the polarization tensor now clarifies the systematics of the underlying physical parameter regimes.

Correspondingly, the photon polarization tensor can be organized in terms of an expansion in the dimensionless quantities $\frac{k_{1} k_{2}}{4 m^{2}}, \lambda$ and $\xi^{2} \lambda^{2}$. In particular, the leading contributions to 
Eq. (5) are of $\mathcal{O}\left(\xi^{2} \lambda^{2}\right)$ and read

$$
\begin{aligned}
G_{1}^{0} & =-G_{2}^{0}=\frac{32}{315} \xi_{1} \xi_{2} \lambda^{2} i \lambda\left(1+\mathcal{O}\left(\frac{k_{1} k_{2}}{4 m^{2}}\right)+\mathcal{O}\left(\lambda^{2}\right)\right), \\
G_{3}^{0} & =-\frac{2}{45}\left(4 \xi_{1}^{2} \lambda^{2}+7 \xi_{2}^{2} \lambda^{2}\right)\left(1+\mathcal{O}\left(\frac{k_{1} k_{2}}{4 m^{2}}\right)+\mathcal{O}\left(\lambda^{2}\right)\right), \\
G_{4}^{0} & =G_{3}^{0}\left(\xi_{1}^{2} \leftrightarrow \xi_{2}^{2}\right) \\
G_{5}^{0} & =-\frac{8}{105} \frac{\sqrt{k_{1}^{2} k_{2}^{2}}}{4 m^{2}}\left(\xi_{1}^{2} \lambda^{2}+\xi_{2}^{2} \lambda^{2}\right)\left(1+\mathcal{O}\left(\frac{k_{1} k_{2}}{4 m^{2}}\right)+\mathcal{O}\left(\lambda^{2}\right)\right),
\end{aligned}
$$

and

$$
\begin{aligned}
G_{1}^{ \pm 1} & =G_{2}^{ \pm 1}= \pm \frac{i}{15} \xi_{1} \xi_{2} \lambda^{2}\left(1+\mathcal{O}\left(\frac{k_{1} k_{2}}{4 m^{2}}\right)+\mathcal{O}\left(\lambda^{2}\right)\right) \\
G_{3}^{ \pm 1} & =\frac{1}{45}\left(4 \xi_{1}^{2} \lambda^{2}-7 \xi_{2}^{2} \lambda^{2}\right)\left(1+\mathcal{O}\left(\frac{k_{1} k_{2}}{4 m^{2}}\right)+\mathcal{O}\left(\lambda^{2}\right)\right) \\
G_{4}^{ \pm 1} & =-G_{3}^{ \pm 1}\left(\xi_{1}^{2} \leftrightarrow \xi_{2}^{2}\right) \\
G_{5}^{ \pm 1} & =\frac{4}{105} \frac{\sqrt{k_{1}^{2} k_{2}^{2}}}{4 m^{2}}\left(\xi_{1}^{2} \lambda^{2}-\xi_{2}^{2} \lambda^{2}\right)\left(1+\mathcal{O}\left(\frac{k_{1} k_{2}}{4 m^{2}}\right)+\mathcal{O}\left(\lambda^{2}\right)\right)
\end{aligned}
$$

whereas the leading contributions to $G_{j}^{l}$ with $|l| \geq 2$ scale as $\sim\left(\xi^{2} \lambda^{2}\right)^{|l|}$ and thus are at least of $\mathcal{O}\left(\left(\xi^{2} \lambda^{2}\right)^{2}\right)$. Plugging these terms into Eqs. (2)-(5), we obtain a compact approximation to the photon polarization tensor for a generic, elliptically polarized plane wave background in the parameter regime where $\left\{\xi^{2} \lambda^{2},|\lambda|,\left|\frac{k_{1} k_{2}}{4 m^{2}}\right|\right\} \ll 1$. The above findings imply that the infinite sum in Eq. (5) at $\mathcal{O}\left(\xi^{2} \lambda^{2}\right)$ receives contributions only for $l= \pm 1$. Hence, the persistent inelastic interactions can be associated with the absorption/release of just two laser photons.

As a particular example, we consider the special case of an incoming on-shell photon with $k_{1}^{\mu}=\omega_{1}\left(1, \vec{k}_{1} /\left|\vec{k}_{1}\right|\right)$, fulfilling $k_{1}^{2}=0$. In this case, the parameter $\lambda$ can be written as $\lambda \rightarrow \frac{\omega \omega_{1}}{2 m^{2}}\left(1-\cos \Varangle\left(\vec{k}, \vec{k}_{1}\right)\right)$, such that

$$
\lambda^{2} \xi^{2} \quad \rightarrow \quad\left(\frac{e \mathfrak{E}}{m^{2}}\right)^{2} \frac{\omega_{1}^{2}}{4 m^{2}}\left(1-\cos \Varangle\left(\vec{\kappa}, \vec{k}_{1}\right)\right)^{2}
$$

where we employed the shorthand notation $\mathfrak{E}^{2} \in\left\{\mathfrak{E}_{1}^{2}, \mathfrak{E}_{2}^{2}, \mathfrak{E}_{1} \mathfrak{E}_{2}\right\}$. Obviously, the dependence on the frequency $\omega$ of the plane wave background drops out and the combination $\lambda^{2} \xi^{2}$ becomes $\omega$ independent. Correspondingly, the photon polarization tensor at $\mathcal{O}\left(\xi^{2} \lambda^{2}\right)$ in the limit $\omega \rightarrow 0$ is obtained straightforwardly in this case: It is given by Eq. (2) with $c_{5}=0$ [see the remarks below Eq. (9)], and the projectors (3) and other coefficients (5) specialized to $\omega=0$. Obviously, it only features an elastic contribution and its coefficients [cf. Eq. (5)] 
are given by

$$
c_{j} \quad \rightarrow \quad i(2 \pi)^{4} m^{2} \frac{\alpha}{\pi} \delta\left(k_{1}-k_{2}\right) \tilde{G}_{j},
$$

with $\left.\tilde{G}_{j} \equiv\left[G_{j}^{0}+G_{j}^{+1}+G_{j}^{-1}\right]\right|_{\omega=0}$ and $j \in\{1, \ldots, 4\}$. Inserting the explicit expressions from Eqs. (18) and (19) into Eq. (21), we obtain $\tilde{G}_{1}=\tilde{G}_{2}=0$ as well as $\tilde{G}_{3}=-\frac{28}{45} \xi_{2}^{2} \lambda^{2}$ and $\tilde{G}_{4}=-\frac{16}{45} \xi_{2}^{2} \lambda^{2}$. As expected the dependence on $\xi_{1}$ completely drops out and the polarization tensor in this limit eventually depends only on the single field strength $\mathfrak{E}_{2}$. Recall that the electromagnetic field components follow by differentiations of the four-vector potential (11), which explains why the electric field $\mathfrak{E}_{2}$, persists even though it comes along with a factor of $\sin (\kappa x)$ in Eq. (11). Putting everything together, we finally obtain

$$
\Pi^{\mu \nu}\left(k_{1}, k_{2}\right) \rightarrow-i(2 \pi)^{4} \delta\left(k_{1}-k_{2}\right) \frac{\alpha}{\pi} \omega_{1}^{2}\left(1-\cos \Varangle\left(\vec{\kappa}, \vec{k}_{1}\right)\right)^{2}\left(\frac{e \mathfrak{E}_{2}}{m^{2}}\right)^{2}\left[\frac{7}{45} \Lambda_{1}^{\mu} \Lambda_{1}^{\nu}+\frac{4}{45} \Lambda_{2}^{\mu} \Lambda_{2}^{\nu}\right] .
$$

This reproduces the photon polarization tensor for constant crossed fields at $\mathcal{O}\left(\left(\frac{e \mathfrak{E}}{m^{2}}\right)^{2}\right)$ and on-the-light-cone dynamics [37, 38].

\section{B. Laser photon merging}

For a given laser photon polarization, i.e., a particular choice of the monochromatic plane wave background (1), the photon merging amplitude depends on both the explicit expression for the field inhomogeneity and the polarization state $\epsilon_{\mu}^{*(p)}(k)$ of the outgoing photon, with $p$ labeling the two transverse photon polarizations. It is given by [39]

$$
\mathcal{M}^{(p)}(k)=\frac{\epsilon_{\mu}^{*(p)}(k)}{\sqrt{2 k^{0}}} \int \frac{\mathrm{d}^{4} q}{(2 \pi)^{4}} \Pi^{\mu \nu}(k, q) A_{\nu}(q)
$$

where $A_{\nu}(q)=\int_{x} \mathrm{e}^{-i x q} A_{\nu}(x)$ is the Fourier transform of the gauge field representing the inhomogeneous electromagnetic field in position space; the star symbol * denotes complex conjugation. The explicit expression for $k^{\mu}=\left(k^{0}, \vec{k}\right)$ depends of course on the specific merging process to be considered. For the merging of $2 n$ laser photons of frequency $\omega$ in a static field, momentum conservation and the fact that the outgoing photon is real and propagates on the light cone imply that $k^{0}=|\vec{k}|=2 n \omega$. Moreover, given this condition, the coefficient $c_{5}$ in Eq. (21) vanishes [cf. below Eq. (91)], such that the tensor structure of $\Pi^{\mu \nu}(q, k)$ can be expressed solely in terms of $\Lambda_{1}^{\mu}$ and $\Lambda_{2}^{\mu}$.

As outlined in detail above, in this article we limit ourselves to the study of the merging process in a static magnetic field. We consider field inhomogeneities of the form $\vec{B}(x)=B(x) \vec{e}_{B}$, such that the direction of the magnetic field $\vec{e}_{B}$ is fixed globally and only 
its amplitude is varied. More specifically, we set $\vec{e}_{B}=\vec{e}_{\mathrm{z}}$ and focus on a one dimensional spatial inhomogeneity in $\mathrm{x}$ direction, i.e., $B(x) \rightarrow B(\mathrm{x})$, such that $\vec{\nabla} B(\mathrm{x}) \sim \vec{e}_{\mathrm{x}}$. The wave vector of the laser photons is assumed to be $\vec{\kappa}=\kappa_{\mathrm{x}} \vec{e}_{\mathrm{x}}+\kappa_{\mathrm{y}} \vec{e}_{\mathrm{y}}$, i.e., the incident laser photons do not have a momentum component parallel to the magnetic field (cf. Fig 3). Even if they had, such a component would not be affected due to translational invariance along the $\mathrm{z}$ direction.

Utilizing $\kappa^{2}=0$ it is convenient to introduce the angle parameter $\theta \in\left[0 \ldots \frac{\pi}{2}\right]$ and write $\kappa^{\mu}=\omega(1, \cos \theta, \sin \theta, 0)$ with $\omega>0$. Correspondingly, the orthogonality relations $a_{1} \kappa=a_{2} \kappa=a_{1} a_{2}=0$ imply that the parametrization of the orthonormal vectors $a_{1}^{\mu}$ and $a_{2}^{\mu}$ just requires one additional angle parameter which we denote by $\varphi \in[0 \ldots 2 \pi)$. We write

$$
\begin{aligned}
& a_{1}^{\mu}=(0,-\sin \theta \cos \varphi, \cos \theta \cos \varphi,-\sin \varphi), \\
& a_{2}^{\mu}=(0,-\sin \theta \sin \varphi, \cos \theta \sin \varphi, \cos \varphi),
\end{aligned}
$$

i.e., our conventions are such that the spatial components of $\kappa^{\mu}, a_{1}^{\mu}$ and $a_{2}^{\mu}$ form a righthanded trihedron (cf. Fig 3 ). The choice of $\theta$ fixes the propagation direction $\vec{\kappa}$ of the incident photons relative to the inhomogeneity, while $\varphi$ controls the orientation of the vectors $\vec{a}_{1}$ and $\vec{a}_{2}$ spanning the spatial subspace transverse to $\vec{\kappa}$.

A convenient choice for the four-vector potential giving rise to a magnetic field of the desired type is

$$
A^{\mu}(x)=A(\mathrm{x}) e_{\mathrm{y}}^{\mu}, \quad \text { with } \quad A(\mathrm{x})=\int^{\mathrm{x}} \mathrm{dx}^{\prime} B\left(\mathrm{x}^{\prime}\right)
$$

where we have defined $e_{\mathrm{y}}^{\mu} \equiv\left(0, \vec{e}_{\mathrm{y}}\right)$. The lower limit of the integral is left unspecified as it does not have any observable consequences and thus can be chosen arbitrarily. Finally, a Fourier transform of Eq. (25) yields the momentum space representation of the four-vector potential as needed in Eq. (23),

$$
A^{\mu}(q)=(2 \pi)^{3} \delta\left(q_{0}\right) \delta\left(q_{\mathrm{y}}\right) \delta\left(q_{\mathrm{z}}\right) A\left(q_{\mathrm{x}}\right) e_{\mathrm{y}}^{\mu}, \quad \text { with } \quad A\left(q_{\mathrm{x}}\right)=\int_{-\infty}^{\infty} \mathrm{dx} \mathrm{e}^{-i \mathrm{x} q_{\mathrm{x}}} A(\mathrm{x}) .
$$

Plugging this expression into Eq. (23) and introducing $\bar{q}^{\mu} \equiv\left(0, q_{\mathrm{x}} \vec{e}_{\mathrm{x}}\right)$, the photon merging amplitude can be simplified significantly and reads

$$
\mathcal{M}^{(p)}(k)=\frac{\epsilon_{\mu}^{*(p)}(k)}{\sqrt{2 k^{0}}} \int \frac{\mathrm{d} q_{\mathrm{x}}}{2 \pi} \Pi^{\mu 2}(k, \bar{q}) A\left(q_{\mathrm{x}}\right) .
$$

Substituting $k_{2} \rightarrow \bar{q}$ into the expressions for $\Lambda_{1}^{\mu}$ and $\Lambda_{2}^{\mu}$ in Eq. (3) we obtain together with 
Eq. (24)

$$
\begin{aligned}
& \Lambda_{1}^{\mu}=\left(\tan \theta \cos \varphi, 0, \frac{\cos \varphi}{\cos \theta},-\sin \varphi\right), \\
& \Lambda_{2}^{\mu}=\left(\tan \theta \sin \varphi, 0, \frac{\sin \varphi}{\cos \theta}, \cos \varphi\right) .
\end{aligned}
$$

Analogously to Eq. (5), we write

$$
\Pi^{\mu 2}(k, \bar{q})=(2 \pi)^{4} \sum_{l \in \mathbb{Z}} \delta(k-\bar{q}-2 l \kappa) \Pi_{l}^{\mu 2}(k, \bar{q})
$$

where the explicit representation

$$
\Pi_{l}^{\mu 2}=i m^{2} \frac{\alpha}{\pi} \frac{1}{\cos \theta}\left[\Lambda_{1}^{\mu}\left(G_{1}^{l} \sin \varphi+G_{3}^{l} \cos \varphi\right)+\Lambda_{2}^{\mu}\left(G_{2}^{l} \cos \varphi+G_{4}^{l} \sin \varphi\right)\right]
$$

makes use of Eq. (28). Using Eq. (29) in Eq. (27), the residual integration over $q_{\mathrm{x}}$ can be performed and we obtain

$$
\mathcal{M}^{(p)}(k)=(2 \pi)^{3} \delta\left(k_{\mathrm{z}}\right) \sum_{l \in \mathbb{Z}} \delta\left(k^{0}-2 l \omega\right) \delta\left(k_{\mathrm{y}}-2 l \omega \sin \theta\right) \frac{\epsilon_{\mu}^{*(p)}(k)}{\sqrt{2 k^{0}}} \Pi_{l}^{\mu 2}(k, \tilde{k}) A\left(\tilde{k}_{\mathrm{x}}\right)
$$

with $\tilde{k}^{\mu} \equiv\left(0,\left(k_{\mathrm{x}}-2 l \omega \cos \theta\right) \vec{e}_{\mathrm{x}}\right)$.

Taking into account the fact that the outgoing photon has positive energy $\left(k^{0}>0\right)$ and propagates on the light cone $\left(k_{\mu} k^{\mu}=0\right)$, and also because of the $\delta$ functions for the y and $\mathrm{z}$ momentum components, we identify $k^{0} \equiv 2 l \omega$ and rewrite the $\delta$ function implementing energy conservation in Eq. (31) as follows,

$$
\delta\left(k^{0}-2 l \omega\right) \rightarrow \delta_{l 0} \delta\left(k_{\mathrm{x}}\right)+\Theta\left(l+0^{+}\right) \frac{1}{\cos \theta}\left[\delta\left(k_{\mathrm{x}}-2 l \omega \cos \theta\right)+\delta\left(k_{\mathrm{x}}+2 l \omega \cos \theta\right)\right],
$$

where $\Theta($.$) is the Heaviside function. Correspondingly, we have$

$$
\begin{aligned}
\mathcal{M}^{(p)}(k)=(2 \pi)^{3} \delta\left(k_{\mathrm{z}}\right) \sum_{l=1}^{\infty} \frac{1}{\cos \theta}\left[\delta \left(k_{\mathrm{x}}-2 l \omega\right.\right. & \left.\cos \theta)+\delta\left(k_{\mathrm{x}}+2 l \omega \cos \theta\right)\right] \\
& \times \delta\left(k_{\mathrm{y}}-2 l \omega \sin \theta\right) \frac{\epsilon_{\mu}^{*(p)}(k)}{\sqrt{4 l \omega}} \Pi_{l}^{\mu 2}(k, \tilde{k}) A\left(\tilde{k}_{\mathrm{x}}\right),
\end{aligned}
$$

with $k^{\mu}=\left(2 l \omega, k_{\mathrm{x}}, k_{\mathrm{y}}, 0\right)$, where we have made use of the fact that the $l=0$ contribution vanishes: it scales $\sim \delta(\vec{k}) \frac{\Pi_{l}^{\mu 2}(k, \tilde{k})}{\sqrt{4 l \omega}} \sim \delta(\vec{k}) l^{3 / 2} \rightarrow 0$ [cf. also Eq. (34) below].

When adapted to the particular kinematics in Eq. (33) (cf. the arguments of the photon polarization tensor), the dimensionless parameters $\frac{k_{1} k_{2}}{4 m^{2}}, \lambda$ and $\xi^{2} \lambda^{2}$ governing the expansion 
of the photon polarization tensor performed in Sec. IIA all vanish for the contribution $\sim \delta\left(k_{\mathrm{x}}-2 l \omega \cos \theta\right)$. For the contribution $\sim \delta\left(k_{\mathrm{x}}+2 l \omega \cos \theta\right)$ they are non-zero and read

$$
\begin{aligned}
\frac{k_{1} k_{2}}{4 m^{2}} \equiv \frac{k \tilde{k}}{4 m^{2}} & \rightarrow \frac{1}{2}\left(\frac{2 l \omega \cos \theta}{m}\right)^{2} \\
\lambda & \rightarrow\left(\frac{2 l \omega \cos \theta}{m}\right) \frac{\omega \cos \theta}{m} \\
\xi^{2} \lambda^{2} & \rightarrow\left(\frac{e \mathfrak{E}}{m^{2}}\right)^{2}\left(\frac{2 l \omega \cos \theta}{m}\right)^{2} \cos ^{2} \theta
\end{aligned}
$$

Neglecting higher-order contributions of $\mathcal{O}\left(\frac{k_{1} k_{2}}{4 m^{2}}\right) \sim \mathcal{O}(\lambda) \sim \mathcal{O}\left(\frac{\omega^{2}}{m^{2}}\right)$, our result will of course be fully governed by the remaining parameters $\xi_{1}^{2} \lambda^{2}, \xi_{2}^{2} \lambda^{2}$ and $\xi_{1} \xi_{2} \lambda^{2}$.

As a result, the number of merged photons with four wave-vector $k_{f}^{\mu}$ and polarization $p$ according to Fermi's golden rule is given by

$$
\mathcal{N}^{(p)}\left(k_{f}\right)=\int \frac{d^{3} k}{(2 \pi)^{3}}\left|\mathcal{M}^{(p)}(k)\right|^{2}=T L_{\mathrm{y}} L_{\mathrm{z}} \sum_{l=1}^{\infty} \frac{\left|\epsilon_{\mu}^{*(p)}\left(k_{f}\right) \Pi_{l}^{\mu 2}\left(k_{f}, \tilde{k}_{f}\right) A\left(\tilde{k}_{f, \mathrm{x}}\right)\right|^{2}}{4 l \omega \cos \theta}
$$

with $k_{f}^{\mu}=2 l \omega(1,-\cos \theta, \sin \theta, 0)$, i.e., the outgoing photon of energy $2 l \omega$ propagates in $(-\cos \theta, \sin \theta, 0)$ direction. Moreover, $\tilde{k}_{f}^{\mu}=-4 l \omega \cos \theta\left(0, \vec{e}_{\mathrm{x}}\right)$ encodes the momentum transfer from the field inhomogeneity, $T$ is the interaction time and $L_{\mathrm{y}} L_{\mathrm{z}}$ is the interaction area transverse to the inhomogeneity. The total number of merged photons is

$$
\mathcal{N}\left(k_{f}\right)=\sum_{p} \mathcal{N}^{(p)}\left(k_{f}\right)
$$

Obviously the dominant contribution is due to the merging of just two laser photons, $l=1$, as higher photon processes are suppressed by at least a factor of $\xi^{2} \lambda^{2}$. Correspondingly, Eq. (33) can be written as

$$
\mathcal{N}^{(p)}\left(k_{f}\right)=T L_{\mathrm{y}} L_{\mathrm{z}} \frac{\left|\epsilon_{\mu}^{*(p)}\left(k_{f}\right) \Pi_{1}^{\mu 2}\left(k_{f}, \tilde{k}_{f}\right) A\left(\tilde{k}_{f, \mathrm{x}}\right)\right|^{2}}{4 \omega \cos \theta}\left(1+\mathcal{O}\left(\frac{e^{2} \mathfrak{E}^{2}}{m^{4}} \frac{\omega^{2}}{m^{2}}\right)\right)
$$

We emphasize that the terms written out explicitly in Eq. (37) account for the entire twophoton merging process. We approximate the infinite sum in Eq. (35) by its contribution for $l=1$, and thereby neglect merging processes of $2 l$ laser photons with $l>1$.

Employing the substitutions $\varphi \rightarrow \varphi^{\prime}$ and $\theta \rightarrow \pi-\theta$ in Eq. (24), we introduce the following two vectors

$$
\epsilon^{(1) \mu}\left(k_{f}\right)=\left(0,-\sin \theta \cos \varphi^{\prime},-\cos \theta \cos \varphi^{\prime},-\sin \varphi^{\prime}\right)
$$




$$
\epsilon^{(2) \mu}\left(k_{f}\right)=\left(0,-\sin \theta \sin \varphi^{\prime},-\cos \theta \sin \varphi^{\prime}, \cos \varphi^{\prime}\right)
$$

with $\varphi^{\prime} \in[0 \ldots 2 \pi)$ fixed, to span the subspace transverse to the wave-vector $\vec{k}_{f}$ of the merged photon. The two polarization degrees of freedom of the outgoing photon are then conveniently expressed in terms of the vectors $\epsilon^{(p) \mu}\left(k_{f}\right)$, with $p \in\{1,2\}$, representing linear polarization states in the particular basis characterized by a particular choice of $\varphi^{\prime}$. Polarizations other than linear can be obtained through linear combinations of the vectors (38).

We are now in a position to provide the explicit expressions of the polarization tensor in Eq. (35) contracted with a given polarization vector of the outgoing photon, which read

$$
\begin{aligned}
\epsilon_{\mu}^{*(1)}\left(k_{f}\right) \Pi_{l}^{\mu 2}=i m^{2} \frac{\alpha}{\pi} \frac{1}{2 \cos \theta}\left[\operatorname { s i n } \varphi ^ { \prime } \left(G_{1}^{l}\right.\right. & \left.-G_{2}^{l}\right)-\sin \left(\varphi^{\prime}+2 \varphi\right)\left(G_{1}^{l}+G_{2}^{l}\right) \\
& \left.-\cos \left(\varphi^{\prime}+2 \varphi\right)\left(G_{3}^{l}-G_{4}^{l}\right)-\cos \varphi^{\prime}\left(G_{3}^{l}+G_{4}^{l}\right)\right],
\end{aligned}
$$

and

$$
\epsilon_{\mu}^{*(2)}\left(k_{f}\right) \Pi_{l}^{\mu 2}=\left.\epsilon_{\mu}^{*(1)}\left(k_{f}\right) \Pi_{l}^{\mu 2}\right|_{\varphi^{\prime} \rightarrow \varphi^{\prime}-\frac{\pi}{2}} .
$$

Introducing the dimensionless field strengths $\varepsilon_{i} \equiv \frac{e \mathfrak{E}_{i}}{m^{2}}$ with $i \in\{1,2\}$, in particular the $l=1$ contribution to Eq. (39) can be written as

$$
\begin{aligned}
\epsilon_{\mu}^{*(1)}\left(k_{f}\right) \Pi_{1}^{\mu 2}=i(\omega \cos \theta)^{2} & \frac{\alpha}{\pi} \frac{2}{15} \cos \theta\left[-2 i \sin \left(\varphi^{\prime}+2 \varphi\right) \varepsilon_{1} \varepsilon_{2}\right. \\
& \left.+\cos \left(\varphi^{\prime}+2 \varphi\right)\left(\varepsilon_{1}^{2}+\varepsilon_{2}^{2}\right)-\frac{11}{3} \cos \varphi^{\prime}\left(\varepsilon_{1}^{2}-\varepsilon_{2}^{2}\right)\right]\left(1+\mathcal{O}\left(\frac{\omega^{2}}{m^{2}}\right)\right),
\end{aligned}
$$

where we have made use of Eqs. (19) and (34).

If $A\left(\tilde{k}_{f, \mathrm{x}}\right)$ is either purely real or imaginary valued, which is true for the field inhomogeneities symmetric in $\mathrm{x}$ to be considered below, the modulus squared can be split and Eq. (37) be represented as follows,

$$
\mathcal{N}^{(p)}\left(k_{f}\right)=T L_{\mathrm{y}} L_{\mathrm{z}} \frac{\left|\epsilon_{\mu}^{*(p)}\left(k_{f}\right) \Pi_{1}^{\mu 2}\left(k_{f}, \tilde{k}_{f}\right)\right|^{2}\left|A\left(\tilde{k}_{f, \mathrm{x}}\right)\right|^{2}}{4 \omega \cos \theta}\left(1+\mathcal{O}\left(\frac{e^{2} \mathfrak{E}^{2}}{m^{4}} \frac{\omega^{2}}{m^{2}}\right)\right)
$$

The modulus squared of Eq. (41) is obtained straightforwardly and reads

$$
\begin{aligned}
\left|\epsilon_{\mu}^{*(1)}\left(k_{f}\right) \Pi_{1}^{\mu 2}\right|^{2}=(\omega \cos \theta)^{4} & \frac{\alpha^{2}}{\pi^{2}} \frac{4}{225} \cos ^{2} \theta\left\{4\left(\varepsilon_{1} \varepsilon_{2}\right)^{2}-\frac{22}{3} \cos \varphi^{\prime} \cos \left(\varphi^{\prime}+2 \varphi\right)\left(\varepsilon_{1}^{4}-\varepsilon_{2}^{4}\right)\right. \\
+ & {\left.\left[\frac{121}{9} \cos ^{2} \varphi^{\prime}+\cos ^{2}\left(\varphi^{\prime}+2 \varphi\right)\right]\left(\varepsilon_{1}^{2}-\varepsilon_{2}^{2}\right)^{2}\right\}\left(1+\mathcal{O}\left(\frac{\omega^{2}}{m^{2}}\right)\right) }
\end{aligned}
$$

while the analogous expression for the other polarization mode $(p=2)$ follows from Eq. (40). 
Aiming at the total number of merged photons in the polarization basis characterized by a particular choice of $\varphi^{\prime}$, we have to add the moduli squared corresponding to the two different polarization states [cf. Eqs. (36) and (42)]. This results in

$$
\begin{aligned}
\sum_{p=1}^{2}\left|\epsilon_{\mu}^{*(p)}\left(k_{f}\right) \Pi_{1}^{\mu 2}\right|^{2} & =(\omega \cos \theta)^{4} \frac{\alpha^{2}}{\pi^{2}} \frac{8}{225} \cos ^{2} \theta \\
& \times\left[4\left(\varepsilon_{1} \varepsilon_{2}\right)^{2}-\frac{11}{3} \cos (2 \varphi)\left(\varepsilon_{1}^{4}-\varepsilon_{2}^{4}\right)+\frac{65}{9}\left(\varepsilon_{1}^{2}-\varepsilon_{2}^{2}\right)^{2}\right]\left(1+\mathcal{O}\left(\frac{\omega^{2}}{m^{2}}\right)\right)
\end{aligned}
$$

which is completely independent of the choice of $\varphi^{\prime}$, as it should. Noteworthily, in case of circularly polarized incident laser photons for which $\xi_{1}=\xi_{2}$ and thus $\varepsilon_{1}=\varepsilon_{2}$, the contributions for both polarization modes individually become independent of $\varphi$ and $\varphi^{\prime}$; cf. Eq. (43). Equation (44) upon insertion into Eq. (36) and accounting for the prefactors displayed in Eq. (37) represent a central result of this work.

Subsequently, we assume the probe laser to deliver incident laser pulses of duration $\tau$, entering under an angle $\theta$ and featuring a circular transverse beam profile. The longitudinal evolution of the probe laser pulses follows the envelope of a Gaussian beam, with beam waist right at the intersection with the field inhomogeneity. We denote the transverse cross-section area at the beam waist by $\sigma$. Correspondingly, the transversal area $L_{\mathrm{y}} L_{\mathrm{z}}$ can be identified with the intersection area of such a beam profile with the $\mathrm{y}-\mathrm{z}$ plane, i.e., $L_{\mathrm{y}} L_{\mathrm{z}}=\frac{\sigma}{\cos \theta}$ (cf. Fig. 41). Assuming that the magnetic field inhomogeneity is long-lived as compared to the pulse duration $\tau$ of the probe laser, it is reasonable to consider $\tau$ as a measure of the interaction time $T$, and set $T=\tau$. Hence, we can make use of the following substitution,

$$
T L_{\mathrm{y}} L_{\mathrm{z}} \rightarrow \frac{\sigma \tau}{\cos \theta}
$$

\section{RESULTS AND DISCUSSION}

Let us now consider explicit examples of localized magnetic field inhomogeneities which can be tackled analytically. We limit ourselves to two elementary shapes, characterized by just two parameters, namely an amplitude $B$ and a typical extension $w$. For a Lorentz profile characterized by its full width at half maximum (FWHM),

$$
B(\mathrm{x})=\frac{B}{1+\left(\frac{2 \mathrm{x}}{w}\right)^{2}},
$$




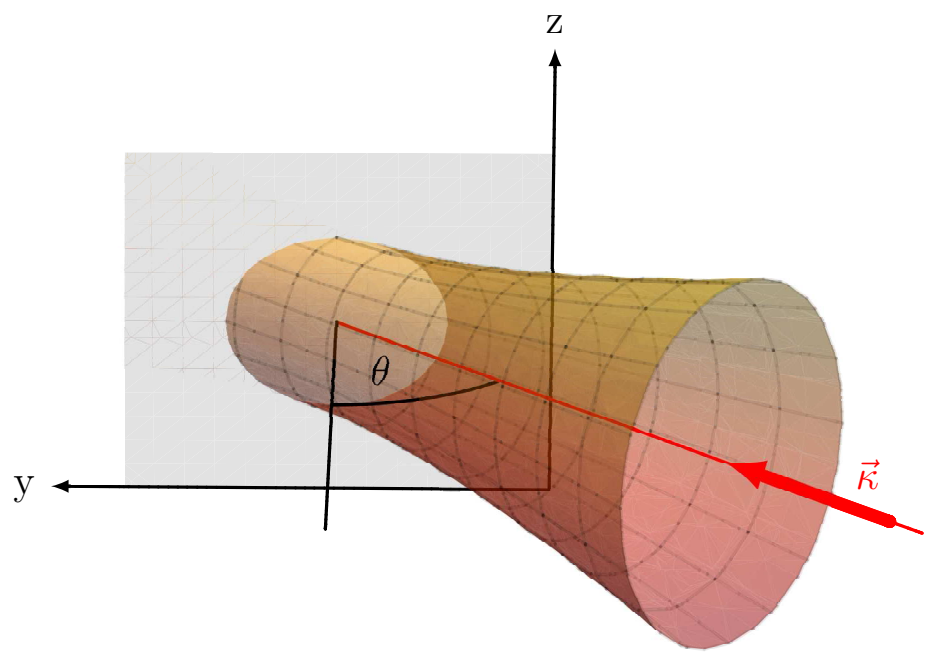

FIG. 4: Sketch of the envelope of a Gaussian beam intersecting the $\mathrm{y}-\mathrm{z}$ plane in the vicinity of its waist under an angle of $\theta$ (cf. also Fig. 3). Given that the transverse cross-section area of the Gaussian beam at the beam waist is a circle of area $\sigma$, the intersection area is an ellipse with area $\frac{\sigma}{\cos \theta}$.

the associated gauge field in position space can be determined by Eq. (25). We obtain

$$
A(\mathrm{x})=\frac{B w}{2} \arctan \left(\frac{2 \mathrm{x}}{w}\right)
$$

and Fourier transforming to momentum space via Eq. (26),

$$
A\left(q_{\mathrm{x}}\right)=-i \frac{\pi B w}{2 q_{\mathrm{x}}} \mathrm{e}^{-\frac{\left|q_{\mathrm{x}}\right| w}{2}}
$$

Analogously, for a Gaussian type inhomogeneity characterized by its full width at 1/e of its maximum,

$$
B(\mathrm{x})=B \mathrm{e}^{-\left(\frac{2 \mathrm{x}}{w}\right)^{2}},
$$

we obtain

$$
A(\mathrm{x})=\frac{\sqrt{\pi} B w}{4} \operatorname{erf}\left(\frac{2 \mathrm{x}}{w}\right),
$$

where erf(.) denotes the error function, and finally

$$
A\left(q_{\mathrm{x}}\right)=-i \frac{\sqrt{\pi} B w}{2 q_{\mathrm{x}}} \mathrm{e}^{-\left(\frac{q_{\mathrm{x}} w}{4}\right)^{2}}
$$

Equations (48) and (151) share an overall prefactor $\sim\left(-i \frac{\sqrt{\pi} B w}{2 q_{\mathrm{x}}}\right)$, but differ in the exponential decay. For the Lorentz profile the decay is linear in $\left|q_{\mathrm{x}}\right|$ w, while for the Gaussian inhomogeneity it is quadratic in this dimensionless parameter. 
It is now straightforward to derive the number of merged laser photons, Eq. (42), for these inhomogeneities. The number of outgoing merged laser photons with polarization $p=1$ and energy $2 \omega$ reads [cf. Eqs. (42) and (45)]

$$
\begin{aligned}
& \mathcal{N}^{(1)}\left(k_{f}\right)=w \sigma \tau(e B)^{2}(\omega w) \frac{\alpha \cos ^{2} \theta}{57600 \pi}\left\{\begin{array}{c}
\mathrm{e}^{-(4 \omega \cos \theta) w} \\
\frac{1}{\pi} \mathrm{e}^{-\frac{1}{8}(4 \omega \cos \theta)^{2} w^{2}}
\end{array}\right\} \\
& \times\left\{4\left(\varepsilon_{1} \varepsilon_{2}\right)^{2}-\frac{22}{3} \cos \varphi^{\prime} \cos \left(\varphi^{\prime}+2 \varphi\right)\left(\varepsilon_{1}^{4}-\varepsilon_{2}^{4}\right)\right. \\
&\left.+\left[\frac{121}{9} \cos ^{2} \varphi^{\prime}+\cos ^{2}\left(\varphi^{\prime}+2 \varphi\right)\right]\left(\varepsilon_{1}^{2}-\varepsilon_{2}^{2}\right)^{2}\right\}\left(1+\mathcal{O}\left(\frac{\omega^{2}}{m^{2}}\right)\right),
\end{aligned}
$$

where the upper line in braces is the result for the Lorentz (46) and the lower line that for the Gaussian (49) profile, and

$$
\mathcal{N}^{(2)}\left(k_{f}\right)=\left.\mathcal{N}^{(1)}\left(k_{f}\right)\right|_{\varphi^{\prime} \rightarrow \varphi^{\prime}-\frac{\pi}{2}}
$$

As the results for the Lorentz and Gaussian inhomogeneities - apart from the different exponential behavior - are of very similar structure, we find it convenient to adopt the twocomponent notation employed in Eq. (52) in the remainder of this paper. Equation (52) exhibits several characteristic dependencies on the involved parameters: as to be expected from the underlying Feynman diagram, the leading order effect is proportional to the square of the plane wave intensity, i.e., $\sim \mathfrak{E}^{4}$, and to the square of the magnetic field $\sim B^{2}$. In particular the latter dependence represents a comparatively strong increase of the effect with an enhancement of the peak magnetic background field. Other typical nonlinear phenomena such as photon scattering off a magnetic field $\sim B^{4}$ or photon splitting $\sim B^{6}$ are more strongly suppressed since the $B$ field scale is measured in terms of the electron mass scale. On the other hand, the inhomogeneous field has to provide the necessary momentum transfer $\sim 4 \omega \cos \theta$, and the effect is exponentially damped with $\sim(4 \omega \cos \theta) w$.

In this respect, it is instructive to compare these expressions with the number of photons experiencing quantum reflection [16] for the very same conditions, i.e., for incident photons of the same energy, angle of incidence and polarization, and exactly the same field inhomogeneities as in Eqs. (46) and (49).

For completeness, we note that in Ref. [16], the field inhomogeneity was not accounted for exactly in the sense that the photon polarization tensor was evaluated a priori in the presence of the magnetic field inhomogeneity, but rather the inhomogeneity was built in $a$ posteriori by resorting to the result for a constant magnetic background field and using the constant-field expressions locally. As argued in detail in [16], such an approach is justifiable for inhomogeneities whose typical scale of variation $w$ is much larger than the Compton 
wavelength $\lambda_{c}$ of the charged virtual particles, i.e., $w \gg \lambda_{c}$. Particularly in quantum electrodynamics (QED), where the virtual particles are electrons, $\lambda_{c} \approx 2 \cdot 10^{-6} \mathrm{eV}^{-1} \approx 3.9 \cdot 10^{-13} \mathrm{~m}$, many field inhomogeneities available in the laboratory can be dealt with along these lines.

Reference [16] identifies two situations for which the calculations become particularly simple, corresponding to special alignments of the incident photons' wave vector $\vec{k}$ and polarization plane, the magnetic field $\vec{B}$, and the direction of the inhomogeneity $\vec{\nabla} B$. The one reconcilable with incident photons of four wave-vector $\kappa^{\mu}=\omega(1, \cos \theta, \sin \theta, 0)$ and $\vec{B} \sim \vec{e}_{\mathrm{Z}}$ is that with polarization vector in the plane spanned by $\vec{\kappa}$ and $\vec{B}$, labeled by $\|$ in [16]. To bring the $\|$ case of [16] and the merging scenario discussed here into full kinematic agreement, we specialize the quantum reflection formulae to $\Varangle(\vec{\kappa}, \vec{B})=\frac{\pi}{2}$ and set $\varphi=\varphi^{\prime}=0, \varepsilon_{1}=0$ and $\varepsilon_{2}=\frac{e \mathfrak{E}}{m^{2}}$ in Eqs. (52) and (53), i.e., we specialize to incident laser photons polarized linearly along $\mathrm{z}$, and look for induced outgoing photons in the same polarization basis. Incidentally, it can be shown straightforwardly that the polarization direction is conserved under these circumstances for quantum reflection (cf. [16]), i.e., the quantum reflected photons are still polarized along $z$, while for laser photon merging the induced outgoing photons are polarized differently, namely their polarization vector lies in the $\mathrm{x}-\mathrm{y}$ plane [cf. Eq. (55) below].

The number of quantum reflected photons $\mathcal{N}_{\text {Qref }}$ is obtained by multiplying the number of incident probe photons $N_{\text {probe }}$ with the adequate reflection coefficient, given in Eqs. (27) and (29) of [16]. In order to allow for a more direct comparison with the merging result, we first rewrite $N_{\text {probe }}$ : The number of incident photons per pulse amounts to the ratio of the pulse energy of the probe laser $\mathcal{E}$ and its frequency $\omega$, i.e., $N_{\text {probe }}=\frac{\mathcal{E}}{\omega}$. The intensity $I_{\text {probe }}$ at the focal spot, which is related to the electric field strength in the focus via $I_{\text {probe }}=\mathfrak{E}^{2}$, is determined by $I_{\text {probe }}=\frac{\mathcal{E}}{\sigma \tau}$. Hence, the number of probe photons can be expressed as $N_{\text {probe }}=\frac{\mathfrak{E}^{2} \sigma \tau}{\omega}$, and - neglecting corrections of $\mathcal{O}\left(\left(\frac{e B}{m^{2}}\right)^{6}\right)-$ we finally obtain

$$
\mathcal{N}_{\text {Qref }}=w \sigma \tau \frac{49 \alpha}{129600 \pi}\left(\frac{e B}{m^{2}}\right)^{4}(e \mathfrak{E})^{2}(\omega w) \frac{1}{\cos ^{2} \theta}\left\{\begin{array}{c}
\frac{1}{4}(1+\omega w \cos \theta)^{2} \mathrm{e}^{-2 \omega w \cos \theta} \\
\frac{1}{2 \pi} \mathrm{e}^{-\frac{1}{4}(\omega w \cos \theta)^{2}}
\end{array}\right\}
$$

For the merging process, Eqs. (52) and (53), the same choice of parameters results in

$$
\mathcal{N}^{(1)}=w \sigma \tau \frac{49 \alpha}{129600 \pi}\left(\frac{e \mathfrak{E}}{m^{2}}\right)^{4}(e B)^{2}(\omega w) \cos ^{2} \theta\left\{\begin{array}{c}
\mathrm{e}^{-4 \omega w \cos \theta} \\
\frac{1}{\pi} \mathrm{e}^{-2(\omega w \cos \theta)^{2}}
\end{array}\right\}\left(1+\mathcal{O}\left(\frac{\omega^{2}}{m^{2}}\right)\right)
$$

while $\mathcal{N}^{(2)}=0$, such that $\mathcal{N}_{\text {merg }} \equiv \mathcal{N}^{(1)}$. Both results exhibit an exponential suppression with exponent $\sim w \omega \cos \theta=w \kappa_{\mathrm{x}}$, with $\kappa_{\mathrm{x}}$ being the momentum component of the incident probe photons in the direction of the inhomogeneity [cf. above Eq. (24)]. The suppression 
is more pronounced for the merging process. This can also be understood intuitively by recalling that the momentum transfer from the inhomogeneity is $\left|2 \kappa_{\mathrm{x}}\right|$ for the process of quantum reflection (cf. [16]), while it is twice as large, namely $\left|4 \kappa_{\mathrm{x}}\right|$, for the merging of two laser photons.

Another important point to notice is that in Eq. (54) the transition to large incidence angles $\theta \lesssim \pi / 2$ provides a convenient handle to damp the exponential suppression while at the same time increasing the overall prefactor, which scales inversely with $\cos ^{2} \theta$. Conversely, in Eq. (55) an analogous increase of the angle of incidence to $\theta \lesssim \pi / 2$ diminishes the overall prefactor $\sim \cos ^{2} \theta$. The ratio of Eqs. (55) and (54) can be derived straightforwardly, and reads

$$
\frac{\mathcal{N}_{\text {merg }}}{\mathcal{N}_{\text {Qref }}} \approx 4\left(\frac{\mathfrak{E}}{B} \cos ^{2} \theta\right)^{2}\left\{\begin{array}{c}
\frac{1}{(1+\omega w \cos \theta)^{2}} \mathrm{e}^{-2 \omega w \cos \theta} \\
\frac{1}{2} \mathrm{e}^{-\frac{7}{4}(\omega w \cos \theta)^{2}}
\end{array}\right\} .
$$

It is governed by just two dimensionless quantities, namely the product $\omega w \cos \theta$, measuring the width $w$ of the inhomogeneity in units of the inverse of the momentum component of the incident photons in $\vec{\nabla} B$ direction, and $\mathfrak{E} / B \cos ^{2} \theta$, i.e., the ratio of the field strength of the probe relative to that of the pump, augmented by an extra factor of $\cos ^{2} \theta$.

It is now natural to ask for the conditions which have to be met such that photon merging dominates quantum reflection, i.e., $\mathcal{N}_{\text {merg }} \geq \mathcal{N}_{\text {Qref }}$. Inserting this condition into Eq. (566), we obtain

$$
\frac{\mathfrak{E}}{B} \cos ^{2} \theta \geq \frac{1}{2}\left\{\begin{array}{c}
|1+\omega w \cos \theta| \mathrm{e}^{\omega w \cos \theta} \\
\sqrt{2} \mathrm{e}^{\frac{7}{8}(\omega w \cos \theta)^{2}}
\end{array}\right\} \geq \frac{1}{2}\left\{\begin{array}{c}
1 \\
\sqrt{2}
\end{array}\right\},
$$

where we made use of the fact that the expression on the right-hand side of the first inequality is bounded from below by its value for $\omega w \cos \theta=0$. The latter condition tells us that for the particular set-up considered here, the yields for photon merging can dominate those for quantum reflection only if the quantity $(\mathfrak{E} / B) \cos ^{2} \theta$ is larger than the numerical bounds given on the rightmost side of Eq. (57).

In Fig. 5, we exemplarily set $\mathfrak{E}=B$ which is a natural choice if all fields are provided by a high-intensity laser system. We investigate the implications of the first inequality in Eq. (57) as a function of $\theta$ and $\omega w$. Obviously, for this choice of the field strengths laser photon merging can only dominate quantum reflection if $\cos \theta \geq \frac{1}{\sqrt{2}} \leftrightarrow \theta \leq 45^{\circ}$ $\left(\cos \theta \geq 2^{-1 / 4} \leftrightarrow \theta \leq 32.7^{\circ}\right)$ for a Gaussian (Lorentzian) inhomogeneity. Qualitatively speaking, the merging process tends to dominate for small angles of incidence $\theta$ and small values of $\omega w$. Equation (56) implies that this region (in the $\theta-\omega w$ plane) can be enlarged by increasing the ratio of $\mathfrak{E} / B$.

So far we only focused on the relative importance of the two effects, but did not provide absolute quantitative estimates. Most obviously, as both effects are suppressed by powers 


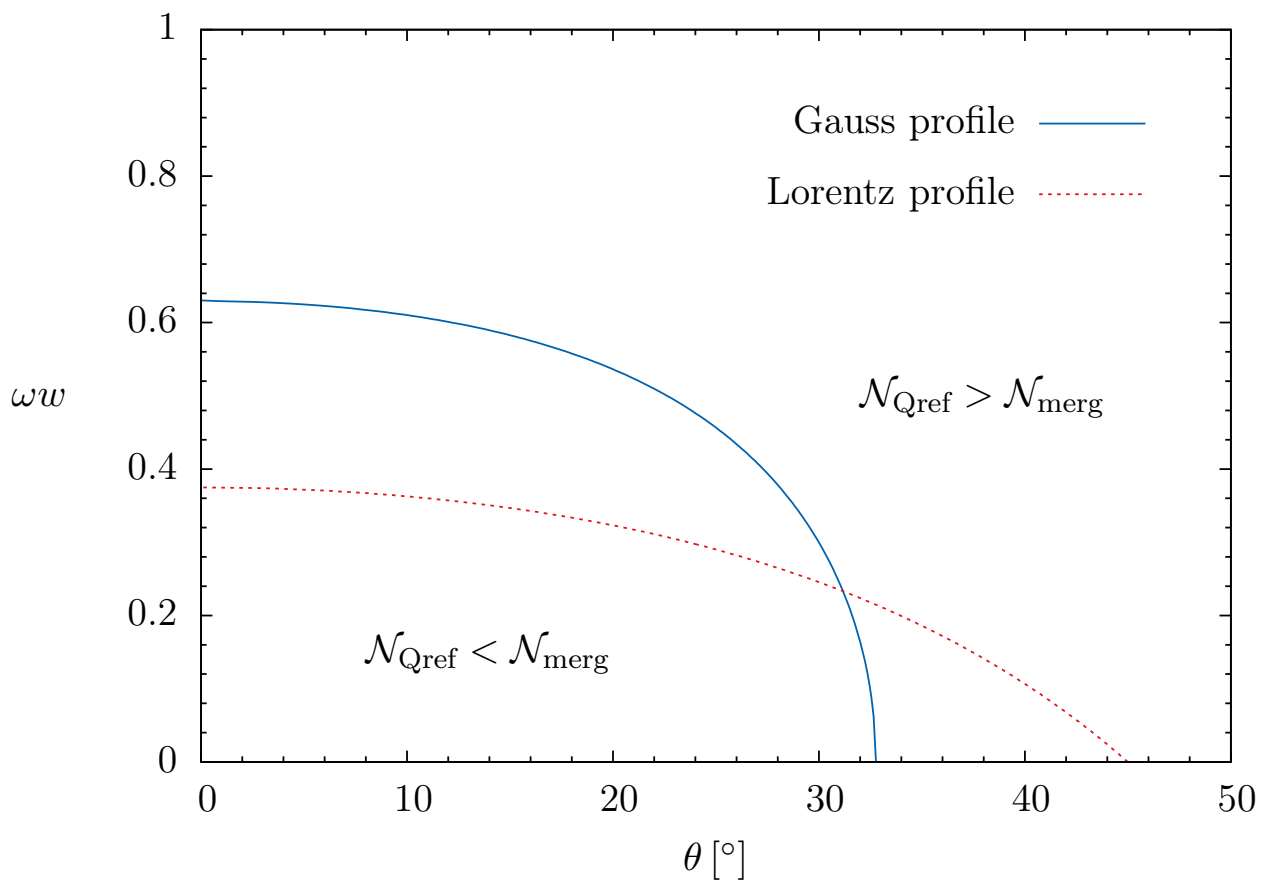

FIG. 5: Choosing $\mathfrak{E}=B$ as an example, we depict the regimes where photon merging dominates quantum reflection and vice versa based on Eq. (57). Photon merging dominates quantum reflection in the regime in the lower left bounded by the blue (solid) and red (dotted) lines for Gauss and Lorentz type inhomogeneities, respectively.

of $\frac{e B}{m^{2}}$ and $\frac{e \mathfrak{E}}{m^{2}}$ [cf. Eqs. (54) and (55)], in order to increase them it is preferable to enlarge the field strengths as much as possible.

Before providing some explicit quantitative estimates, let us briefly discuss the generic features of Eq. (55) and confront it with Eq. (54). Consider the first derivative of the number of merged photons (55) with respect to $w \cos \theta$,

$$
\frac{d \mathcal{N}_{\text {merg }}}{d(w \cos \theta)} \approx \frac{2 \mathcal{N}_{\text {merg }}}{w \cos \theta}\left\{\begin{array}{c}
1-2 \omega w \cos \theta \\
1-2(\omega w \cos \theta)^{2}
\end{array}\right\} \stackrel{!}{=} 0 \quad \rightarrow \quad w \cos \theta=\frac{1}{2 \omega}\left\{\begin{array}{c}
1 \\
\sqrt{2}
\end{array}\right\}
$$

Taking into account the sign of the second derivative, we find that the number of outgoing merged photons has a maximum as a function of $w \cos \theta$ for the above values and reads

$$
\left.\mathcal{N}_{\operatorname{merg}}\right|_{\max } \approx \frac{\sigma \tau}{\omega} \frac{49 \alpha}{129600 \pi}\left(\frac{e \mathfrak{E}}{m^{2}}\right)^{4}(e B)^{2} \frac{1}{4}\left\{\begin{array}{c}
\mathrm{e}^{-2} \\
\frac{2}{\pi} \mathrm{e}^{-1}
\end{array}\right\}
$$

Hence, keeping $w$ fixed, the number of merged photons increases monotonically as a function of $\theta$ from its value for $\theta=0$ until it reaches a maximum at $\theta=\arccos \left(\frac{1}{2 \omega w}\right)$ in case of the Gaussian, and $\theta=\arccos \left(\frac{1}{\sqrt{2} \omega w}\right)$ for the Lorentz type inhomogeneity. Increasing $\theta$ 
even further, it decreases rapidly until it reaches $\mathcal{N}_{\text {merg }}=0$ at $\theta=90^{\circ}$.

Conversely, for fixed $\omega$ the number of quantum reflected photons (54) exhibits a monotonic increase throughout the interval from $\theta=0$ to $\theta=90^{\circ}$. Actually, $\mathcal{N}_{\text {Qref }}$ even diverges for $\theta \rightarrow 90^{\circ}$ due to the cosine squared term in its denominator, an unphysical feature which can be attributed to the unphysical limit of an infinitely long interaction of the probe photons and the inhomogeneity at "grazing incidence" $\theta \rightarrow 90^{\circ}$.

Finally, we provide some rough estimates on the numbers of merged and quantum reflected photons attainable in an all optical pump-probe experiment based on high-intensity lasers. Even though we have just focused on a one-dimensional field inhomogeneity, as in [16] we exemplarily adopt the design parameters of the two high-intensity laser systems to become available in Jena [40]: JETI 200 [41] $\left(\lambda=800 \mathrm{~nm} \approx 4.06 \mathrm{eV}^{-1}, \mathcal{E}=4 \mathrm{~J} \approx 2.50 \cdot 10^{19} \mathrm{eV}\right.$, $\left.\tau=20 \mathrm{fs} \approx 30.4 \mathrm{eV}^{-1}\right)$ as probe, and POLARIS [42] $\left(\lambda_{\text {pump }}=1030 \mathrm{~nm} \approx 5.22 \mathrm{eV}^{-1}\right.$, $\left.\mathcal{E}_{\text {pump }}=150 \mathrm{~J} \approx 9.36 \cdot 10^{20} \mathrm{eV}, \tau_{\text {pump }}=150 \mathrm{fs} \approx 228 \mathrm{eV}^{-1}\right)$ as pump. This is meant to give a first order of magnitude estimate of the number of induced outgoing photons. Let us emphasize that it is certainly a rather crude approximation to adopt the formula derived for a stationary, one-dimensional magnetic field inhomogeneity of Gaussian type (49) to mimic the field inhomogeneity as generated in the focal spot of a high-intensity laser. Such an approximation ignores the longitudinal modulation and evolution of the pump laser pulse. A more rigorous and refined treatment in the context of an all optical pump-probe experiment would require us to account also for the temporal structure and evolution of field inhomogeneities. Fully accounting for pulse shape dependencies has become a subject of increasing importance in strong-field phenomenology with high-intensity lasers. Progress has already been made, for instance, for the case of vacuum birefringence [43, 44].

In generic high-intensity laser experiments the focal spot area cannot be chosen at will, but is limited by diffraction. Assuming Gaussian beams, the effective focus area is conventionally defined to contain $86 \%$ of the beam energy $\left(1 / e^{2}\right.$ criterion for the intensity). The minimum value of the beam diameter in the focus is given by twice the laser wavelength multiplied with $f^{\#}$, the so-called $f$-number, defined as the ratio of the focal length and the diameter of the focusing aperture [45]; $f$-numbers as low as $f^{\#}=1$ can be realized experimentally. Thus, assuming both probe and pump lasers to be focused down to the diffraction limit, the attainable field strengths are of the order of

$$
\mathfrak{E}^{2}=I_{\text {probe }} \approx \frac{0.86 \mathcal{E}}{\tau \sigma}, \quad B^{2}=2 I_{\text {pump }} \approx 2 \frac{0.86 \mathcal{E}_{\text {pump }}}{\tau_{\text {pump }} \sigma_{\text {pump }}},
$$

with $\sigma \approx \pi \lambda^{2}$ and $\sigma_{\text {pump }} \approx \pi \lambda_{\text {pump }}^{2}$. The additional factor of two in the definition of $B$ accounts for the fact that, focusing on a purely magnetic field inhomogeneity, the entire 
laser intensity is considered to be available in terms of a magnetic field, as could, e.g., be realized by superimposing two counter propagating laser beams.

In the most straightforward experimental setting to imagine, the pump laser beam propagates along the y axis, while its transversal profile, parametrized by the coordinate x, evolves along the well-defined envelope of a Gaussian beam, and in the vicinity of the beam waist is to be understood as constituting the Gaussian field inhomogeneity (49) of width $w \approx 2 \lambda_{\text {pump. }}$.

For beams focused down to the diffraction limit, the Rayleigh length is given by the wavelength of the beam multiplied with a factor of $\pi$ [45], i.e., for the pump, $z_{\mathrm{R}}=\pi \lambda_{\text {pump}}$. Hence, over distances of the order of several wave lengths $\lambda_{\text {pump }}$ about the beam waist, the beam diameter remains approximately constant along $\vec{e}_{\mathrm{y}}$ and an experimental setting resembling Fig. 3 is conceivable.

In Fig. 6, we plot the number of induced outgoing photons for both effects as a function of $\theta$. The respective results are obtained straightforwardly by plugging the design parameters of the Jena high-intensity laser systems JETI 200 and Polaris given above into Eq. (60) and the lower components of Eqs. (154), (55) and (59).

Obviously, for this particular all-optical experimental setup the photon merging process is substantially suppressed in comparison with quantum reflection. As detailed below Eq. (55), the differences observed in Fig. 6 can be attributed to the different scaling of Eqs. (54) and (55) with $\cos ^{2} \theta$. While quantum reflection receives an overall enhancement with $\sim \frac{1}{\cos ^{2} \theta}$ for large angles of incidence $\theta \lesssim 90^{\circ}$, photon merging becomes maximal if the condition (59) is met (for the JETI 200 - Polaris setup this is the case for an angle of $\theta \approx 87^{\circ}$, wherefore $\left.\mathcal{N}_{\text {merg }}\right|_{\max } \approx 1.3 \cdot 10^{-3}$ ) and dies off to zero for $\theta \rightarrow 90^{\circ}$.

In practice, an all-optical setup designed to benefit from the geometric noise reduction will work at a reflection angle near or somewhat above $\theta \simeq 80^{\circ}$. For parameters similar to the ones studied here, photon merging then is clearly a negligible background to the quantum reflection signal. Nevertheless, because of its different polarization and frequency dependence, appropriate filtering techniques could still render photon merging detectable in the long run.

\section{CONCLUSIONS AND OUTLOOK}

In this paper we have studied laser photon merging in the presence of a one dimensional, stationary magnetic field inhomogeneity. We have in particular confronted the number of outgoing merged photons with the number of quantum reflected photons for the same conditions and discussed in detail the similarities and differences of the two effects. Sticking to the design parameters of the high-intensity laser facilities to be available in Jena, consisting 


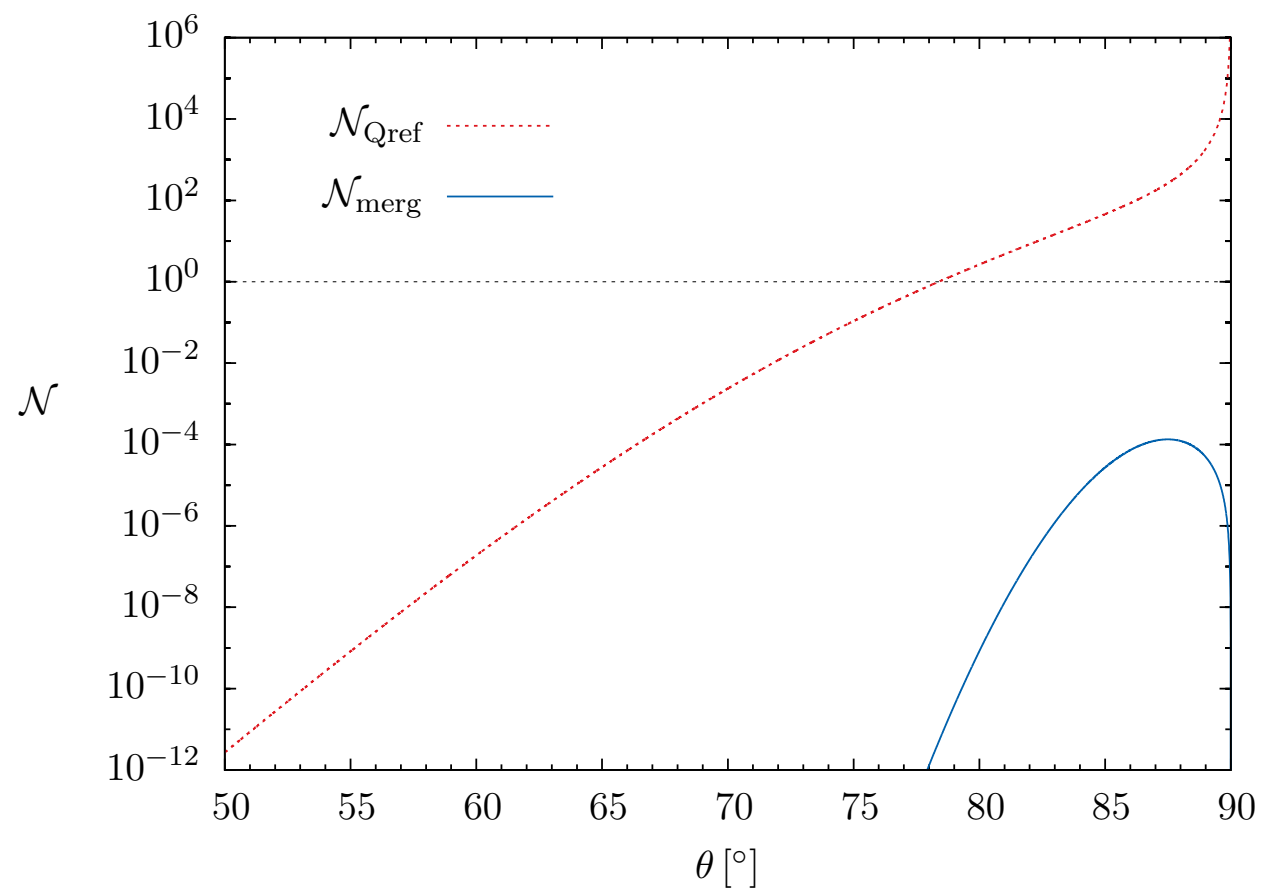

FIG. 6: Number of induced outgoing photons per shot $\mathcal{N}_{\text {merg }}$ due to the effects of laser photon merging and quantum reflection as a function of $\theta$, adopting the design parameters of the Jena highintensity laser systems, JETI 200 and Polaris (cf. main text). The horizontal dashed line shows where the number of induced outgoing photons per shot becomes one. For quantum reflection this is the case for $\theta \geq 78^{\circ}$ [16]. Conversely, the number of outgoing merged photons reaches a maximum at $\theta \approx 87^{\circ}$ and stays below $\left.\mathcal{N}_{\text {merg }}\right|_{\max } \approx 1.3 \cdot 10^{-4}$ throughout the interval $0 \leq \theta \leq 90^{\circ}$; cf. Eq. (59) for the Gaussian inhomogeneity and the discussion below. For completeness, we note that $\left.\mathcal{N}_{\text {Qref }}\right|_{\theta=0} \approx 3 \cdot 10^{-29}$ while $\left.\mathcal{N}_{\text {merg }}\right|_{\theta=0} \approx 3 \cdot 10^{-228}$.

of a petawatt and a terawatt class laser system, we have provided a first rough estimate of the number of merged photons to be potentially attainable in an all-optical experiment. Our results confirm that the quantum reflection signal is a most promising candidate for the discovery of quantum vacuum nonlinearities under controlled laboratory conditions with high-intensity lasers. In particular, it dominates photon merging in a wide parameter range.

The expression for the photon merging number is determined most straightforwardly from the photon polarization tensor in a plane wave background. Actually, the main difficulty in determining the number of outgoing merged laser photons is the problem of finding a convenient and controllable expansion of the photon polarization tensor, allowing us to represent our results in concise expressions. This has led us to adopt a novel expansion strategy to obtain analytical insights into the photon polarization in plane wave backgrounds. We believe that this representation will also be useful in many other strong field physics questions beyond the merging process.

Of course, a natural extension of our present study in the future would be the investigation 
of the photon merging process in more generic, time-dependent inhomogeneities. Such a study is necessary to allow for definitive answers about the the numbers of outgoing merged photons attainable in the focal spot of high-intensity lasers, taking into account the full longitudinal evolution of the pump laser pulse.

\section{Acknowledgments}

We are particularly indebted to Maria Reuter for creating Figs. 3 and 4 . FK is grateful to Matt Zepf for many interesting and enlightening discussions. HG acknowledges support by the DFG under grants Gi 328/5-2 (Heisenberg program) and SFB-TR18. RS acknowledges support by the Ministry of Education and Science of the Republic of Kazakhstan.

[1] W. Heisenberg and H. Euler, Z. Phys. 98, 714 (1936), an English translation is available at physics/0605038.

[2] V. Weisskopf, Kong. Dans. Vid. Selsk., Mat.-fys. Medd. XIV, 6 (1936).

[3] J. S. Schwinger, Phys. Rev. 82, 664 (1951).

[4] J. S. Toll, Ph.D. thesis, Princeton Univ., 1952 (unpublished).

[5] R. Baier and P. Breitenlohner, Act. Phys. Austriaca 25, 212 (1967); Nuov. Cim. B 47117 (1967).

[6] Z. Bialynicka-Birula and I. Bialynicki-Birula, Phys. Rev. D 2, 2341 (1970).

[7] S. L. Adler, Annals Phys. 67, 599 (1971).

[8] G. Cantatore [PVLAS Collaboration], Lect. Notes Phys. 741, 157 (2008); E. Zavattini et al. [PVLAS Collaboration], Phys. Rev. D 77, 032006 (2008) arXiv:0706.3419 [hep-ex]]; F. Della Valle, U. Gastaldi, G. Messineo, E. Milotti, R. Pengo, L. Piemontese, G. Ruoso and G. Zavattini, arXiv:1301.4918 [quant-ph].

[9] P. Berceau, R. Battesti, M. Fouche and C. Rizzo, Can. J. Phys. 89, 153 (2011); P. Berceau, M. Fouche, R. Battesti and C. Rizzo, Phys. Rev. A, 85, 013837 (2012) arXiv:1109.4792 [physics.optics]]; A. Cadene, P. Berceau, M. Fouche, R. Battesti, C. Rizzo, arXiv:1302.5389 [physics.optics].

[10] T. Heinzl, B. Liesfeld, K. -U. Amthor, H. Schwoerer, R. Sauerbrey and A. Wipf, Opt. Commun. 267, 318 (2006) hep-ph/0601076.

[11] W. Dittrich and H. Gies, Springer Tracts Mod. Phys. 166, 1 (2000).

[12] M. Marklund and J. Lundin, Eur. Phys. J. D 55, 319 (2009) [arXiv:0812.3087 [hep-th]].

[13] G. V. Dunne, Eur. Phys. J. D 55, 327 (2009) arXiv:0812.3163 [hep-th]]. 
[14] A. Di Piazza, C. Muller, K. Z. Hatsagortsyan and C. H. Keitel, Rev. Mod. Phys. 84, 1177 (2012) arXiv:1111.3886 [hep-ph]].

[15] B. Marx, et al., Opt. Comm. 284, 915 (2011).

[16] H. Gies, F. Karbstein and N. Seegert, New J. Phys. 15, 083002 (2013) arXiv:1305.2320 [hep$\mathrm{ph}]$.

[17] B. King, A. Di Piazza and C. H. Keitel, Nature Photon. 4, 92 (2010) arXiv:1301.7038 [physics.optics]]; Phys. Rev. A 82, 032114 (2010) [arXiv:1301.7008 [physics.optics]].

[18] D. Tommasini and H. Michinel, Phys. Rev. A 82, 011803 (2010) arXiv:1003.5932 [hep-ph]].

[19] K. Z. Hatsagortsyan and G. Y. Kryuchkyan, Phys. Rev. Lett. 107, 053604 (2011).

[20] Z. Bialynicka-Birula, Physica D 2, 513-524 (1981).

[21] A. E. Kaplan, and Y. J. Ding, Phys. Rev. A 62, 043805 (2000).

[22] S. R. Valluri, U. D. Jentschura and D. R. Lamm, AIP Conf. Proc. 687, 203 (2003) hep-ph/0308223.

[23] A. M. Fedotov and N. B. Narozhny, Phys. Lett. A 362, 1 (2007) hep-ph/0604258.

[24] M. Marklund and P. K. Shukla, Rev. Mod. Phys. 78, 591 (2006) hep-ph/0602123.

[25] A. Di Piazza, K. Z. Hatsagortsyan and C. H. Keitel, Phys. Rev. D 72, 085005 (2005).

[26] A. Di Piazza, K. Z. Hatsagortsyan and C. H. Keitel, Phys. Rev. Lett. 100, 010403 (2008) arXiv:0708.0475 [hep-ph]].

[27] A. Di Piazza, K. Z. Hatsagortsyan and C. H. Keitel, Phys. Rev. A 78, 062109 (2008) arXiv:0906.5576 [hep-ph]].

[28] J. Lundin, M. Marklund, E. Lundström, G. Brodin, J. Collier, R. Bingham, J. T. Mendonça, and P. Norreys, Phys. Rev. A 74, 043821 (2006).

[29] B. King and C. H. Keitel, New J. Phys. 14, 103002 (2012) arXiv:1202.3339 [hep-ph]].

[30] Y. Monden and R. Kodama, Phys. Rev. Lett. 107, 073602 (2011).

[31] B. Döbrich and H. Gies, JHEP 1010, 022 (2010) [arXiv:1006.5579 [hep-ph]].

[32] V. N. Baier, A. I. Milshtein and V. M. Strakhovenko, Zh. Eksp. Teor. Fiz. 69, 1893 (1975) [Sov. Phys. JETP 42, 961 (1976)].

[33] W. Becker and H. Mitter, J. Phys. A: Math. Gen. 81638 (1975).

[34] S. Meuren, C. H. Keitel and A. Di Piazza, Phys. Rev. D 88, no. 1, 013007 (2013) arXiv:1304.7672 [hep-ph]].

[35] F. Karbstein, Phys. Rev. D 88, no. 8, 085033 (2013) arXiv:1308.6184 [hep-th]].

[36] I. S. Gradshteyn and I. M. Ryzhik, Table of Integrals, Series, and Products, Fifth Edition, Academic Press, UK (1994).

[37] N. B. Narozhnyi, Zh. Eksp. Teor. Fiz. 55, 714 (1968) [Sov. Phys. JETP 28, 371 (1969)].

[38] V. I. Ritus, Ann. Phys. 69, 555 (1972).

[39] V. P. Yakovlev, Zh. Eksp. Teor. Fiz. 51, 619 (1966) [Sov. Phys. JETP 24, 411 (1967)].

[40] cf. the homepage of the Helmholtz Institute Jena: http://www.hi-jena.de 
[41] M. Zepf, private communication.

[42] M. Hornung, et. al., Opt. Lett. 38, 718-720 (2013); M. Hornung, et. al., High Power Laser Science and Engineering, vol. 7 (2014), accepted for publication.

[43] A. Di Piazza, K. Z. Hatsagortsyan and C. H. Keitel, Phys. Rev. Lett. 97, 083603 (2006) hep-ph/0602039].

[44] V. Dinu, T. Heinzl, A. Ilderton, M. Marklund and G. Torgrimsson, arXiv:1312.6419 [hep-ph]. V. Dinu, T. Heinzl, A. Ilderton, M. Marklund and G. Torgrimsson, arXiv:1405.7291 [hep-ph].

[45] A. E. Siegman, Lasers, First Edition, University Science Books, USA (1986). 